\title{
Die internationale Zusammenarbeit im Ansturm auf die afrikanischen Länder südlich der Sahara
}

Annie Chéneau-Loquay and Raphaël Ntambue-Tshimbulu

\section{CpenEdition}

\section{Journals}

Electronic version

URL: http://journals.openedition.org/sjep/528

DOI: $10.4000 /$ sjep.528

ISSN: 1663-9677

\section{Publisher}

Institut de hautes études internationales et du développement

\section{Printed version}

Date of publication: 1 novembre 2003

Number of pages: $47-80$

ISSN: $1660-5926$

\section{Electronic reference}

Annie Chéneau-Loquay und Raphaël Ntambue-Tshimbulu, « Die internationale Zusammenarbeit im Ansturm auf die afrikanischen Länder südlich der Sahara », Schweizerisches Jahrbuch für Entwicklungspolitik [Online], 22-2 | 2003, Online erschienen am: 10 Juni 2010, abgerufen am 08 September 2020. URL : http://journals.openedition.org/sjep/528 ; DOI : https://doi.org/10.4000/sjep. 528 


\title{
Die internationale Zusammenarbeit im Ansturm auf die afrikanischen Länder südlich der Sahara
}

\author{
Annie Chéneau-Loquay* \\ Raphaël Ntambue-Tshimbulu**
}

D ie Zusammenarbeit mit den afrikanischen Ländern südlich der Sahara im Bereich der Informations- und Kommunikationstechnologien (IKT) erfolgt in einem Umfeld, in dem die Kosten und Verfahren für die Umsetzung der Projekte zur Integration der Telematiknetze die lokalen finanziellen und technischen Kapazitäten übersteigen und sowohl ein Eingreifen von aussen, als auch eine afrikanische Beteiligung erfordern. Die Jahre internationaler Zusammenarbeit, die in dieser Studie auf den Zeitraum von 1989 bis heute begrenzt wurden, ergeben eine mässige Weiterentwicklung der Visionen und Strategien der beteiligten Akteure, die Stärkung der internationalen Organisationen im Bereich der globalen Bewirtschaftung der IKT, eine starke Zunahme der IKT-Anwendungen auf verschiedenen Gebieten der Entwicklungszusammenarbeit sowie eine schwierige Anpassung all dieser Instrumente an die neuen Herausforderungen des Informationszeitalters.

Vom Norden her betrachtet, in einem Kontext, der dem Markt auf Kosten der Hilfe den Vorzug gibt und in den IKT die Möglichkeit sieht, Entwicklungsetappen zu überspringen, ist die Zusammenarbeit von den spezifischen Interessengebieten der Geber - über die Priorität für die globalen Infrastruktureinrichtungen im Informationsbereich, die Partnerschaft zwischen internationalen Organisationen der Entwicklungszusammenarbeit und privaten Firmen des Nordens - zu einer umfassenderen Vision des „,digitalen Grabens“ übergegangen. Diese Konzeption hat jedoch die internationale Entwicklungshilfepolitik, die mehr den öffentlichen Sektor mit dem privaten und dem Vereinssektor verbindet, nicht signifikant beeinflusst.

Von Afrika aus betrachtet, in einem Kontext der „Hilfemüdigkeit“ und der Definition des Internets als Bedarf für die lokale Entwicklung, ist die Entwicklungszusammenarbeit von der blossen Durchführung der Projekte aus dem Norden zur Diversifizierung der lokalen Erfahrungen vornehmlich gemäss den westlichen Visionen der Geldgeber und dem internationalen Konsens übergegangen.

* Forschungsleiterin am CNRS (Centre national de recherche scientifique), Gestalterin des pluridisziplinären Programms und Forschungsnetzes Africa'nti, l'observatoire des modes d'insertion, des usages et des impacts des technologies de la communication en Afrique et dans les Suds, am CEAN (Centre d'études d'Afrique noire) des Institut d'études politiques in Bordeaux (Frankreich). Siehe: $<$ www.africanti.org >.

** Assoziierter Forschungsleiter am CNRS, CEAN (UMR IEP-CNRS). 
Somit hat sich dieses Konzept der Zusammenarbeit weder von der ,geistigen Hegemonie“ der Entwicklungshelfer des Nordens, noch von der relativen Passivität und der „minimalistischen IKT-Konzeption“ der afrikanischen Partner gelöst. Die Zusammenarbeit wird immer mehr als „Lektionen erteilend“, statt als „sich an der Beseitigung der lokalen Zwänge beteiligend“ empfunden. Die Beispiele der häufig auftretenden Probleme der Infrastruktureinrichtungen, der Förderung der freien Software, des Fernunterrichts und der Forschung in Afrika lassen die ausgetretenen Pfade der Entwicklungszusammenarbeit erkennen.

\section{Einführung}

Die Zusammenarbeit auf dem Gebiet der Informations- und Kommunikationstechnologien (IKT) und vor allem des Internets ist durch eine starke Abweichung zwischen dem Diskurs und der konkreten Praxis vor Ort gekennzeichnet. Dies gilt besonders für den afrikanischen Kontinent. Gleichwohl ist der IKTBereich Gegenstand zahlreicher Empfehlungen und eines weitgehenden Konsenses zur Überwindung des „digitalen Grabens“ und zur „Förderung des Wissenszugangs für alle" gewesen - insbesondere in einer Zeit, in der sich die Vorbereitungen für den Weltgipfel der Vereinten Nationen über die Informationsgesellschaft häufen und eine Welle von Empfehlungen nach sich zieht, welche mehr von den Menschen des Nordens als von der Bevölkerung des Südens ausgehen.

Warum kommt der IKT-Thematik soviel Ehre $\mathrm{zu}^{1}$ ? Die Gründe sind in der intensiven Förderung zu suchen, die durch die internationalen und nationalen Instanzen - von einigen Erfolgsautoren aufgegriffen - in Übereinstimmung mit der Politik der reichen Länder erfolgt. An ihrer Spitze die Vereinigten Staaten, die seit Mitte der 90er Jahre das Paradigma einer "Weltinformationsgesellschaft" geschaffen haben, an der jedes Land und jeder Mensch, ungeachtet seiner wirtschaftlichen und kulturellen Merkmale, für sein Wohl teilhaben sollte. Die grundlegenden Zielsetzungen einer solchen Förderung sind wirtschaftlicher und politischer Art und beruhen auf dem Kultursubstrat einer westlichen Hegemonie. Es geht darum, die Märkte für die Produkte und Dienstleistungen im Kommunikations-, Informations- und Wissensbereich durch eine erzwungene Liberalisierung zu öffnen. Die Öffnung wird durch die internationalen Finanzinstitutionen (Weltbank und IWF) orchestriert und von den WTO-Abkommen abgestützt, wobei der Wettbewerb unter den Betreibern für die armen Länder als das beste Mittel angesehen wird, sich auszurüsten und ,ihren Rückstand aufzuholen“.

In diesem Rahmen befindet sich Afrika aufgrund seiner Gegebenheiten in einer Position der Schwäche, und der Einsatz der Entwicklungszusammenarbeit war nötig, um zu vermeiden, dass sich der Kontinent noch weiter ,marginalisiert“. Welches sind nun aber Umfang, Art und Auswirkungen dieser Zusammenarbeit in einem Umfeld, in dem die öffentliche Entwicklungshilfe beträchtlich zurückgegangen ist? Welches sind die derzeitigen Vorgehensweisen und Praktiken?

1 Dies ist die Frage, die im „Forum für das andere Mali“ (Forum pour l'autre Mali) gestellt wird: $<$ www.autremali.org $>$. 
Wie hoch ist der Beherrschungsgrad der lokalen Akteure - Staaten, Gebietskörperschaften und Zivilgesellschaft - bei der Anwendung der IKT, die als wahres „Sesam öffne dich“ zur Entwicklung bezeichnet werden? Wie gross ist die Wirksamkeit dieser Zusammenarbeit und welches ist - weitergehend - ihr Einfluss?

Es ist heute, nach nur einigen Jahren der Umsetzung (und noch mehr im beschränkten Rahmen eines Artikels) unmöglich, all diese Fragen zu beantworten $^{2}$. Deshalb geht es hier mehr darum, das Schwergewicht auf einige Kritikpunkte zu legen (mit der Gefahr, zuweilen schematisch zu wirken), und zwar ausgehend von zwei Gesichtspunkten: demjenigen der „Entwickler und Entwicklungshelfer" und einem anderen, der mehr das afrikanische Umfeld widerspiegelt.

\section{Eine Vision des Nordens}

Die entwickelten Länder des Nordens spielen, direkt oder über die internationalen Organisationen, bei der Ausarbeitung von Empfehlungen zu den Beziehungen zwischen IKT und Entwicklung eine Antriebsrolle. Sie sind die Akteure der Entwicklungszusammenarbeit in einem Kontext, der heute dem Markt auf Kosten der Hilfe den Vorzug gibt.

\section{Eine Konzeption, die sich scheinbar weiterentwickelt...}

Der Standpunkt der Länder des Nordens als Förderer der Entwicklungszusammenarbeit hat sich weiterentwickelt, indem er von einer allein den Infrastruktureinrichtungen im Telekommunikationsbereich eingeräumten Priorität, wie sie 1984 im Maitland-Bericht „The Missing Link“ (Das fehlende Glied) befürwortet wird, zu der Idee überging, dass es mit der Entwicklung des Internets möglich wäre, Etappen zu überspringen, und danach zu einer umfassenderen Vision des ,digitalen Grabens“" gelangte, welche auch die soziokulturellen Aspekte mit einbezieht und fortan auf eine „neue Partnerschaft“ setzt. Jedoch bleibt weiterhin die klassische lineare Ideologie bestehen, die den technologischen Fortschritt mit dem sozialen Fortschritt verbindet. In welcher Form?

Die Integration der IKT in den Entwicklungsprozess in Afrika geht auf die 70er Jahre zurück, wo sich bereits zwei wesentliche Probleme gestellt hatten: das Problem der Nutzung der elektronischen Datenverarbeitung ${ }^{3}$ auf der Ebene der Staaten und für den Bedarf der nationalen Planung, und das allgemeinere Problem, die Staaten mit einer EDV-Technik für die Entwicklung auszustatten. Um uns aber auf die Zeit der Konvergenz von Informatik, Telekommunikation und

2 Einige Antworten auf diese Fragen sind in unseren folgenden Studien zu finden: A. Chéneau-Loquay (Dir), Enjeux des technologies de la communication en Afrique. Du téléphone à Internet, Paris, Regards-Karthala, 2000, 406 S.; R. Ntambue-Tshimbulu, L'Internet, son web et son e-mail en Afrique. Approche critique, coll. Etudes africaines, Paris, L'Harmattan, 2001, 356 S. Siehe auch: $<$ www.africanti.org $>$.

3 Vgl. Colloque international „Informatique et société“, Paris, 24.-28. September 1979, in Actes du colloque, Band 4, La Documentation française, 1980. Die gleichen Ideen findet man im UNO-Bericht 2458 XXIII vom 20. Dezember 1968 : „Coopération internationale en vue de l'utilisation des ordinateurs et techniques de calcul pour le développement“. 
audiovisueller Technik zu beschränken, sagen wir, dass die Integration der IKT in die Entwicklungsprojekte in Afrika Ende der 80er Jahre beginnt, anlässlich der Ausbreitung der vor der Einführung des Internets bestehenden Netze, die dem Kommunikationsbedarf der auf dem afrikanischen Kontinent verstreuten Forschungszentren mit ihren Direktionsabteilungen in den Metropolen entsprachen. In diesem Zusammenhang sind das französische Projekt für das EDVNetz „Réseau informatique de l'ORSTOM“ (RIO), das zum Computernetz „Réseau intertropical d'ordinateurs“ wurde, dann ab 1998 „Appui au développement des réseaux du Sud“ (ADR), der Abidjan-Knoten des Europäischen Universitäts- und Forschungsnetzes EARN (European Academic and Research Network) und die Entwicklung des Satellitennetzes Healthnet im Dienste der Gesundheit in Afrika zu erwähnen ${ }^{4}$.

Diese anfänglichen Strategien zur Integration der IKT in Afrika waren nicht mit einer globalen Vision der Entwicklung des Kontinents und der Verbesserung der bestehenden Infrastruktur verbunden. Jedes Projekt war ein einzelnes gesondertes Vorhaben und die Entwicklung der IKT ging nicht über den beschränkten Rahmen der Spezialbereiche wie Forschung, Epidemiologie, usw. - kurz gesagt, über die spezifischen Interessengebiete der Geber - hinaus. Dies wird sich 1994 mit der weltweiten Verbreitung der Web-Technologie ändern, die indem sie sich eines Kleincomputerparks bediente, der Privatpersonen bereits zur Verfügung stand - das Internet der breiten Öffentlichkeit zugänglich machte. Dies zwang die internationalen Organisationen, globale Politiken festzulegen, welche fähig waren, die Entwicklungsländer in den Prozess des Erwerbs und der Nutzung der IKT einzubeziehen, und geeignet, Mittel des Nordens für Einsätze im Süden aufzubringen.

Genau gesagt, 1994 auf der Konferenz von Buenos Aires wird die Rede des USVizepräsidenten $\mathrm{Al}$ Gore mit der Idee, eine ,globale Informationsinfrastruktur“ zu schaffen, zur Referenz. Sie setzt den Schwerpunkt auf die Erhöhung der Teledichte, die Reglementierung zur Förderung der Privatinvestitionen und den elektronischen Handel. Es ist eine Zeit der Euphorie, in der die Weltbank und die UNCTAD die Vorstellung des „Technologiesprungs“ und der Möglichkeit für die ärmsten Länder verbreiten, dank der fortgeschrittensten Technologien die „Etappen zu überspringen“. Die Organisation eines G-7-Gipfels 1996 in Midrand (Südafrika) zum Thema „Informationsgesellschaft und Entwicklung“" bringt neben den sieben wohlhabendsten Ländern rund dreissig Länder des Südens sowie die wichtigsten internationalen Organisationen zusammen. Das von Nelson Mandela festgesetzte Ziel ist, „die Entwicklungsländer in die neue Welt der Information einzuführen“, und dieses Treffen lässt die Hoffnung aufkommen, dass „die reichsten Länder der Erde“ sich in grossen Infrastrukturarbeiten engagieren. Die bevorzugte Methode ist jedoch die Heranziehung des Marktes statt der internationalen Hilfe. Die Entwicklung des Internet-Netzwerks in Afrika ist direkt mit den Aufforderungen zur Liberalisierung der historischen Telekommunikationsbetreiber verbunden, um die Infrastruktureinrichtungen, die ein wesentlicher Faktor für die Förderung des öffentlichen Internet-Zugangs sind, auszudehnen und zu verbessern. Dennoch ist der Fortschritt hinsichtlich Anschlüsse und Nutzungen enttäuschend.

4 Siehe : <www.rio.net> und <www.healthnet.org $>$. 
Der G-8-Gipfel in Okinawa im Juni 2000 zeigt eine weitere Perspektivenänderung auf. Er bezieht erstmals Vertreter der Zivilgesellschaft und der Unternehmen mit ein, um das Bestehen eines sich zwischen armen und reichen Ländern vertiefenden „digitalen Grabens“ festzustellen. Eine Arbeitsgruppe wird geschaffen, um Lösungen zur Überwindung des Grabens vorzuschlagen. Die Empfehlungen dieser Gruppe (Digital Observatory Task Force, DOT) behandeln die Frage in ihren verschiedenen Aspekten und rufen zur Zusammenarbeit und zu neuen Finanzierungsmethoden in Form von Besteuerungen auf. Jedoch finden die von der Zivilgesellschaft vorgebrachten Empfehlungen nur wenig Anklang.

Die Informations- und Kommunikationstechnologien werden fortan im Zusammenhang mit den internationalen Strategien zur Bekämpfung der grossen Übel der Entwicklungsländer, wie Armut, Verfall der Erziehungs- und Gesundheitssysteme, Analphabetismus, Auflösung der klassischen Kommunikationsnetze gegenüber den modernen Netzen, mit der gesellschaftlichen Organisation verbundene Ungleichheiten, usw., wahrgenommen. Es geht darum, diese Technologien überall dort zu entwickeln, wo sie zur Stärkung der Kapazitäten im Kampf gegen die Unterentwicklung beitragen, besonders auf den verschiedenen Gebieten der Zusammenarbeit, in den Bereichen Wirtschaft, Gesundheit, Erziehung, lokale Organisationen, Verwaltung, usw.

Um dies zu erreichen, wird verstärkt Bezug auf eine neue Partnerschaft genommen, insbesondere auf dem Millenniums-Gipfel der Vereinten Nationen im September 2000. Dort wird im letzten Teil der Ziele empfohlen, eine Weltpartnerschaft für die Entwicklung durch die IKT zu bilden. Der Aktionsplan von Genua, der im Juli 2001 von der G-8 angenommen wurde, wie auch der Aktionsplan für Afrika, der ebenfalls von der G-8 auf dem Gipfel in Kananaskis (Kanada) im Juni 2002 verabschiedet wurde, gingen in die gleiche Richtung; die Teilnehmer nahmen einen Aktionsplan für Afrika als Rahmen für die von den afrikanischen Staatsoberhäuptern konzipierte Initiative „Neue Partnerschaft für die Entwicklung Afrikas" NEPAD ${ }^{6}$ (New Partnership for African Development) an und verpflichteten sich, die öffentliche Entwicklungshilfe bis zum Jahr 2006 um jährlich 12 Milliarden Dollar zu erhöhen.

Bedeutet diese Option der Partnerschaft einen Wandel der Entwicklungshilfepolitik, die mehr den öffentlichen Sektor mit dem privaten Sektor verbinden würde, oder die Fortsetzung der gegenwärtigen Tendenzen, das heisst die Abnahme der öffentlichen Entwicklungshilfe ${ }^{7}$ seit Anfang der 90er Jahre zugunsten des freien Verkehrs von Milliarden Dollar Privatkapital auf der Suche nach rentablen Anlagen?

\footnotetext{
Aktionsplan der G-8 für Afrika: <www.g8.gc.ca/2002Kananaskis/kananaskis/afraction-fr.pdf>.

Siehe weiter unten.

Eine interessante Studie über die öffentliche Entwicklungshilfe im Zusammenhang mit Afrika findet sich in Fr. Gaulme, „Les nouvelles orientations de l'aide publique au développement“, Afrique contemporaine, Sondernummer, 4. Quartal 1998, S. 101-118; J.A. Michel, Coopération pour le développement. Efforts et politiques des membres du Comité d'aide au développement. Rapport du président du Comité d'aide au développement, Ausgabe 1999, Paris, OCDE, 1999. Die öffentliche Entwicklungshilfe, die eine der wichtigsten Finanzierungsquellen der Entwicklungsländer darstellt, machte
} 
Die Entwicklungszusammenarbeit im IKT-Bereich spielt sich heute in einem Umfeld der Anfechtung der traditionellen Formen technischer Zusammenarbeit $\mathrm{ab}$, das von der Vorrangstellung des liberalen Einheitsdenkens geprägt wird, für welches die Nord-Süd-Beziehungen nicht mehr als finanzieller Beitrag, sondern als Freihandelslogik gemäss der Devise „Trade not aid“ (Handel statt Hilfe) verstanden werden, die aber in der Frage abweicht, welche Rolle der öffentlichen Gewalt einzuräumen sei ${ }^{8}$.

Der Internationale Währungsfonds (IWF) hat Wirtschaftstheorien entwickelt, die zeigen, dass die Erhöhung der öffentlichen Entwicklungshilfe für die sehr armen Länder mehr mikro- und makroökonomische Probleme mit sich bringen als lösen würde 9 . Für die Weltbank ist die Hilfe nur in den Ländern nützlich, wo „gute“ Wirtschaftspolitik gemacht wird und „effiziente“ Institutionen ${ }^{10}$ bestehen, (mit dem stillschweigenden Zusatz) welche die Handelsgeschäfte erleichtern. Desgleichen festigt sich das Dogma, gemäss dem die Hilfe zwar die Investitionen, aber nicht den Betrieb unterstützen soll. Im Rahmen dieser Konzeption kommen neue Tendenzen auf, zu denen die Zusammenarbeit im IKT-Bereich gehört.

Heutzutage geht der Finanzierungsbedarf der Entwicklungsprojekte in den afrikanischen Ländern südlich der Sahara immer mehr mit einer Entpolitisierungserscheinung einher, die darin besteht, dem Staat seine Legitimität abzusprechen und ihm die „Zivilgesellschaft“ gegenüberzustellen. Man ,wendet die Unternehmensführung auf jede Organisationsform (Mikrokredite, Bauernverbände, informeller Sektor, Bezirkskomitee) an ; man denkt in Begriffen wie Effizienz, Wirkung, und nicht in politischen Begriffen. Die Entwicklung wird immer häufiger vor allem als wirtschaftliches - bestenfalls als soziales, aber gewiss nicht als politisches Problem angesehen, das heisst als Macht, die durch alle Gruppen, einschliesslich der ärmsten, geht ${ }^{\text {"11 }}$.

im Jahr 2000 lediglich 0,27\% des BSP der reichen Länder aus, trotz der ergebnislos gebliebenen Empfehlung der UNCTAD II, auf Vorschlag des Pearson-Berichts, 1\% des BSP der reichen Länder zu erzielen sowie der Empfehlung Frankreichs unter Mitterrand und derjenigen der Vereinten Nationen, die öffentliche Entwicklungshilfe auf 0,7\% des BSP anzuheben. Eine kritische Studie zur öffentlichen Entwicklungshilfe findet sich in J.-M. Severino, „Les fondements de l'aide au développement au XXI ${ }^{\mathrm{e}}$ siècle“, Critique internationale, Januar 2001.

8 Weitere Argumente sind zu finden in B. Buyck, Emploi par la Banque de l'assistance technique pour le développement institutionnel, Document de travail des services de la Banque mondiale, $\mathrm{n}^{\circ}$ 578, Washington, D.C., BIRD, 1991; UNDP, Technical Cooperation in African Development: An Assessment of Its Effectiveness on Support of the UN Program of Action for African Economic Recovery and Development, 1986-1990, PANUREDA, Washington, D.C., 1990; J. Gaillard, R. Waast, „Quelles politiques de coopération scientifique et technique avec l'Afrique ?“, Afrique contemporaine, Sondernummer, 4. Quartal 1998, S. 85-97.

9 Vgl. P.S. Heller, S. Gupta, „Les enjeux de l'expansion des flux d'aide“, Finances et développement (Vierteljahreszeitschrift des IWF), Juni 2002, <www.imf.org/pubs/ft/fandd/fre/2002/06>. Heller ist stellvertretender Direktor der Abteilung für öffentliche Finanzen des IWF und Gupta zweiter Direktor der gleichen Abteilung.

10 Siehe die Reflexion über die Wirksamkeit der Hilfe im Bericht der Weltbankgruppe Assessing Aid (1999). Die Selektivität der Hilfe erscheint dort als eine Neuerung: die Hilfe ist für die ,verdienstvollen“ Länder bestimmt, die Ratschläge für die „verdienstlosen“ Länder.

11 Levy, M., „Gouttes d'huile ou grains de sable“, in Voyage dans la galaxie des ONG. Actes du colloque sur les ONG et leur rôle dans la coopération au développement, Brüssel, 2. Oktober 1999, Demain le monde, Januar 2000, S. 4. 
Unter dem gleichen Gesichtspunkt besteht eine andere starke Tendenz in der fortschreitenden Aufwertung der dezentralisierten Zusammenarbeit, der Kooperation zwischen kommunalen Körperschaften, die in den Begriffen "Gebiet“ und „lokale Aktion“ denkt.

Hingegen berücksichtigt die sektorielle Zusammenarbeit ${ }^{12}$, so wie sie von der Europäischen Union nach ihrer Einführung 1980 durch die Weltbank konzipiert und zu Sector Wide Approach (SWAp) umbenannt ist, die Entwicklung auf nationaler Ebene und für einen gesamten Sektor (wie Bildungs-, Gesundheitswesen, usw.) oder einen Untersektor (beispielsweise Wasserstrassennetz). Sie bevorzugt Strategien globaler Aktion, die sich in einer Politik und einem Ausgabenprogramm niederschlagen. Sie legt besonderen Nachdruck auf die aktive Rolle des Empfängerstaates bei der Gestaltung und Umsetzung der Zusammenarbeit, was dazu tendieren würde, die Rolle des Staates wieder positiver einzuschätzen, aber eher als technischer Akteur, denn als politische Einheit, die strategische Entscheidungen zu treffen hätte. Gleichwohl wird in den UN-Instanzen, besonders beim UNDP und bei der Wirtschaftskommission für Afrika (CEA), auch immer mehr über die Einführung nationaler IKT-Strategien gesprochen.

Eine weitere Problematik, die man als „globalisierend“ bezeichnen kann, wurde in Frankreich im Jahr 2001 vom Hohen Rat für internationale Zusammenarbeit (Haut Conseil de la coopération internationale, HCCI) ${ }^{13}$, der dem Premierminister zur Seite steht, in den Vordergrund gestellt. Die Problematik der „globalen öffentlichen Güter" (biens publics mondiaux, BPM) wurde als neuer Ansatz diskutiert, der die Politik der internationalen Zusammenarbeit neu zu begründen fähig ist, indem sie die Geldgeber und die Entwicklungshilfeorganisationen auf Probleme weltweiter Konnotation ausrichtet, das heisst auf Fragen, welche sowohl die Länder des Nordens als auch des Südens betreffen (bzw. betreffen können) und die Interdependenz der Völker sowie den gegenseitigen Nutzen hervortreten lassen. Diese Problematik neigt dazu, den Begriff der Hilfe als „Unterstützung“ abzulehnen, indem sie die Vorstellung entwickelt, dass die Länder des Südens paradoxerweise trotz ihrer Armut einige Schlüssel für die Zukunft der ganzen Welt in den Händen halten. Hieraus ergibt sich die Notwendigkeit der „Mitentwicklung“ (codéveloppement), für welche die gegenseitigen Vorteile der Zusammenarbeit klar festgestellt sind. Dies gilt vor allem dann, wenn man das Internet zu einem, ,globalen öffentlichen Gut" macht.

\section{Eine Einbeziehung aller internationalen Akteure entsprechend wesentlicher Ausrichtungen}

Alle grossen internationalen Organisationen, UN-Institutionen oder Organisationen für regionale Zusammenarbeit haben - ungeachtet ihrer Eigenkonzep-

12 Für weitere Informationen siehe M.-P. Crozet, D. Schmid, „L'approche sectorielle: une nouvelle modalité de l'aide européenne au développement“, Afrique contemporaine, Nr. 193, 1. Quartal 2000, S. 76-89.

13 Siehe HCCI, Biens publics mondiaux et coopération internationale. Nouvelle stratégie pour de nouveaux enjeux, Paris, Karthala, 2002, 328 S.; I. Kaul, I. Grunberg, M.A. Stern (dir.), Les biens publics à l'échelle mondiale. La coopération internationale au XXIe siècle, Oxford, Oxford University Press, 1999. 
tion - ihre Politik unter dem Gesichtspunkt einer den IKT einzuräumenden Priorität überprüft, jedoch üben die Nichtregierungsorganisationen (NRO) und die „Zivilgesellschaft“ des Nordens in diesem Bereich ebenfalls einen nicht unwesentlichen Einfluss aus.

Auf bilateraler Ebene haben mehrere Länder finanzielle Mittel für die Förderung der Entwicklungszusammenarbeit mit Afrika auf dem Gebiet der IKT bereitgestellt.

Ab 1996 hat die Leland-Initiative von USAID in Anwendung der Idee einer globalen Infrastruktur im Informationsbereich rund zwanzig Ländern Internet-Verbindungen durch ein Programm in Höhe von 15 Millionen Dollar angeboten. Das kanadische Forschungszentrum für internationale Entwicklung (CRDI) hat mit dem Acacia-Konzept, das zum Zeitpunkt der Konferenz von 1996 über die „Informations- und Entwicklungsgesellschaft“ - der ersten, die in einem Entwicklungsland (Südafrika) stattfand - Gestalt angenommen hatte, eine Pionierrolle gespielt. Das CRDI stand somit von Anfang an mit den Bemühungen afrikanischer Länder in enger Verbindung. Im März 1997 billigte der Gouverneursrat des CRDI die Acacia-Initiative, die darauf abzielt, das Potenzial der Informations- und Kommunikationstechnologien zu ermitteln, um den armen afrikanischen Gemeinschaften Macht zu übertragen. Frankreich mit seinem Entwicklungsforschungsinstitut (Institut de recherche pour le développement, IRD) und seinem Programm RIO (Réseau intertropical d'ordinateurs) hat ebenfalls eine bahnbrechende Rolle gespielt, indem es seine Forschungszentren in Afrika 1989 mit dem Zentrum in Montpellier verband, sodann seine Netzwerke auf andere Forschungszentren ausdehnte und sie an das entstehenden Internet-Netzwerk anschloss. Danach hat Frankreich durch seinen Aussenminister ein prioritäres Kooperationsprogramm für die IKT (PROCOPTIC) eingerichtet, das 1997 beschlossen wurde und sich auf 30 Millionen Francs belief. Dieses Programm stellte den NRO und den Forschungsprogrammen Mittel zur Verfügung, um die Herstellung von Inhalten und Anwendungen, die Schulung im Bereich der neuen Technologien, die Hilfe zur Niederlassung lokaler Führungskräfte im juristischen Bereich und die Entwicklung öffentlicher InternetZugangsstellen für Afrika zu unterstützen.

Der Kanton Genf hat sich seinerseits der Regierung von Mali angenähert, indem er eine bilaterale Zusammenarbeit durch das Anais-Netz ${ }^{14}$ entwickelte, das nach dem Treffen zum Thema „Afrika und die neuen Informationstechnologien“ (Genf, 17. bis 18. Oktober 1996) gebildet wurde, um die Internet-Anwendungen in der Zivilgesellschaft zu fördern. Genf stand hinter der Initiative der BamakoTreffen 2000 und 2002. Bamako 2000 brachte über tausend Teilnehmer aus NRO-Kreisen zusammen, hauptsächlich um zu ermitteln, wie das Internet als Brücke zur Entwicklung dienen kann. Bamako 2002 war die erste Vorbereitungskonferenz für den auf Dezember 2003 anberaumten Weltgipfel über die Informationsgesellschaft.

Die Zusammenarbeit dehnt sich heute auf die Partnerschaft mit den asiatischen Ländern aus, wie der Afrika-Asien-Workshop über die IKT vom 25. bis 29.

$14<$ <ww.anais.org>. 
März 2002 in Kuala Lumpur betonte. Dies bezeugt auch das Projekt „TICAD IT Initiative"15, das auf die Errichtung eines elektronischen Zentrums für den Austausch von Handelsinformationen zwischen Afrika und Asien (in Benin) sowie auf die Organisation eines Workshops in Cotonou zum Thema „Zusammenarbeit zwischen Afrika und Asien und öffentlich-private Partnerschaft in den Strategien zur Entwicklung der IKT in Afrika" ausgerichtet ist.

Die Zusammenarbeit mit den afrikanischen Ländern südlich der Sahara im IKTBereich führte zu einer völlig neuen Partnerschaft zwischen einer internationalen Entwicklungsorganisation, im vorliegenden Fall das UN-Entwicklungsprogramm (UNDP), und einer amerikanischen multinationalen Firma des Privatsektors, Cisco Systems, dem weltweit führenden Unternehmen im Bereich der Netztechnologien für das Internet. Diese Partnerschaft wird über eine Stiftung (NETAID) abgewickelt, die zum obersten Ziel hat, zur Armutsbekämpfung beizutragen. Dies ist eine andere Art für diese Firma, ihre Vorherrschaft zu behaupten und stellt ein Beispiel einer neuen geheimen Absprache zwischen öffentlichem und privatem Sektor unter dem Vorwand eines humanitären Einsatzes dar. Die Partnerschaft führte zu Ausbildungszentren mit Diplomabschluss, sog. „Cisco-Akademien“ die in über 147 Ländern verbreitet sind, davon in mindestens 19 afrikanischen Ländern ${ }^{16}$.

Doch die Hauptakteure der Entwicklungszusammenarbeit im IKT-Bereich sind heutzutage die internationalen Organisationen. Sie spielen eine immer stärkere Rolle bei der Gouvernanz dieses Sektors, indem sie Initiativen an Stelle der schwächelnden Staaten ergreifen, und diese anspornen. Die Weltbank ist heute das wichtigste multilaterale Finanzierungsinstrument der Entwicklungsländer; sie spielt ausserdem für diese Länder eine Katalysatorrolle bei den öffentlichen und privaten Finanzierungen ${ }^{17}$. Sie hat die Kommunikationstechnologien an die Spitze ihrer Prioritäten gestellt. Die UNESCO hat das regionale EDV-Netzwerk für Afrika geschaffen, und das UN-Entwicklungsprogramm (UNDP) führte das Programm „Internet in Afrika“ ein. Das Programm unterstützt die Ausarbeitung nationaler Strategien im IKT-Bereich. Die Organisation intergouvernementale

15 TICAD steht für „Internationale Konferenz in Tokio über die Entwicklung Afrikas“. Es handelt sich dabei um eine regionale Initiative für Afrika, die 1993 mit der gemeinsamen Unterstützung der japanischen Regierung, der UNO und der Weltkoalition für Afrika (Coalition mondiale pour l'Afrique, CMA) durchgeführt wurde. Sie umfasst die afrikanischen und asiatischen Länder, die als Entwicklungspartner für Afrika engagierten Geberländer, die internationalen Organisationen und die Zivilgesellschaft. Die TICAD wacht darüber, dass die Frage der Entwicklung Afrikas weiterhin im Vordergrund steht, sie regt einen hochrangigen Dialog über zentrale Themen an, mobilisiert die Unterstützung für die prioritären Entwicklungsbereiche in Afrika und schlägt ein Leitschema für Massnahmen vor, um Aktionen von gegenseitigem Interesse durchzuführen.<www.ticad.gouv.bj>.

16 Siehe Cisco-Akademien unter der Adresse: <www.cisco.com>. Wir kommen weiter unten auf diese „Akademien“ zurück.

17 In seinem Bericht an die französische Nationalversammlung stellt sich der Abgeordnete Yves Tavernier die Frage, ob ,die zahlreichen Initiativen der Bank, welche zumeist amerikanische Vorschläge aufgreifen, nicht ein Weg sind, um die Vereinten Nationen ihrem Einfluss zu unterwerfen. Die Vereinigten Staaten kontrollieren die Bank, nicht die Vereinten Nationen, welche den Entwicklungsländern eine bedeutende Rolle einräumen“ (Nationalversammlung, Rapport d'information déposé par la Commission des finances, de l'économie générale et du plan sur les activités et le contrôle du Fonds monétaire international et de la Banque mondiale présenté par M. Yves Tavernier, député, 12. Dezember 2000, S. 240). 
de la francophonie (OIF) gründete den Fonds francophone des inforoutes, und die Agence interuniversitaire de la francophonie schuf virtuelle Hochschulcampus, nachdem sie Anfang der 90er Jahre beim Anschluss der Universitäten ans Internet durch das Bildschirmtextgerät (Minitel) eine Pionierrolle gespielt hatte. Viele andere Fonds waren an der Organisation von Konferenzen und Schulungstagungen über die IKT beteiligt. Die internationale Zusammenarbeit hat auch neue Formen von IKT-Initiativen entwickelt. Diese Projekte bilden vor allem technische und soziale Experimente und sind Erfolgsbedingungen unterworfen, um auf den gesamten afrikanischen Kontinent ausgedehnt zu werden. Eine dieser Initiativen ist unter dem Konzept „gemeinschaftliche Mehrzweck-Telezentren“ (télécentres communautaires polyvalents, TCP) oder „gemeinschaftliche Zugangsstellen“ bekannt. Das TCP-Projekt in Timbuktu (Mali) stellt ein historisches Modell dar, das mehrere internationale Akteure wie die FAO, die UNESCO, die ITU, die WHO und das kanadische Forschungszentrum CRDI mobilisiert hat.

Man stellt seit zwei Jahren eine Stärkung derjenigen internationalen Organisationen fest, die damit beauftragt sind, sich weltweit für eine bessere Nutzung des Internets und für die Überbrückung des digitalen Grabens einzusetzen. Sie versuchen, die internationalen Bemühungen für die Integration der IKT in die Entwicklungsprojekte in Afrika zu kanalisieren. Die Expertengruppe der G-8 über den Zugang zu den neuen Technologien (GEANT) sowie die Arbeitsgruppe der UNO über die IKT (GTNU-TIC) wollen Hauptakteure bei der Erschliessung der Möglichkeiten der Informations- und Kommunikationstechnologien für die globale Entwicklung sein. Sie wirken als Katalysatoren und spielen eine politische Führungsrolle bei der Mobilisierung der internationalen Gemeinschaft für das gemeinsame Ziel, am Informationszeitalter teilzunehmen. Sie versuchen, sämtliche weltweiten Initiativen auf gemeinsame Punkte zusammenstreben zu lassen, um eine Kohärenz der Politiken und eine Koordination bei der Umsetzung der IKT-Initiativen zu gewährleisten. In Bezug auf den Aktionsplan von Genua ist die Expertengruppe (GEANT) in mehrere Projekte für Afrika einbezogen - wie unter anderem die Initiative der französischen Regierung zur Unterstützung der digitalen Erschliessung des französischsprachigen Afrikas („Aide au désenclavement numérique de l'Afrique francophone“, ADEN) mit einem Budget von 6,5 Millionen US-Dollar, das Projekt zur Förderung des IKT-Zugangs in Afrika („Catalyser l'accès aux TIC en Afrique“, CATIA) oder das Projekt zur Unterstützung der Telezentren (,Service d'entraide et de dépannage des télécentres") - welche eine Stärkung der Kapazitäten im IKT-Bereich im Dienst der Gesundheit, Erziehung, Kultur usw. anstreben. Die im Jahr 2001 lancierte weltweite Initiative Global Internet Policy Initiative umfasst bereits ein afrikanisches Land, Nigeria, und arbeitet ihrerseits an der Errichtung eines Regulierungsrahmens, der einer optimalen und zunehmenden Nutzung des Internets förderlich ist. Die Neue Partnerschaft für die Entwicklung Afrikas (NEPAD) setzt - trotz ihrer da und dort angemerkten Lücken - ihre Überwachung und Betreuung der afrikanischen Ziele im IKTBereich durch ihre afrikanische Kommission über die neuen Technologien fort. Letztere erhielt vor kurzem ihre Akkreditierung bei der internationalen Gemeinschaft, die sich intensiv mit der IKT-Frage beschäftigt. 
Neben den institutionellen Akteuren der Entwicklungszusammenarbeit werden im IKT-Bereich wie auch in den anderen Bereichen die westlichen Nichtregierungsorganisationen tätig, die bei der Entwicklung der vor dem Internet bestehenden Netzwerke vor allem im englischsprachigen Afrika - APC, Healthnet - bahnbrechend waren, und die jetzt in verschiedener Form, je nach den sie interessierenden Bereichen - Gemeinschaftszugang, Schulung, ländliche Entwicklung, Gesundheitswesen - entweder durch direkte Einsätze oder mittels einer Partnerschaft vorgehen. So zum Beispiel CSDPTT (Frankreich) und Yam Pukri (Burkina Faso), wo ein Mitglied von CSDPTT im Juni und Juli 2002 ,freiwillig“ die von Yam Pukri organisierte Einführung in die IKT übernahm; oder auch die Unterstützung des französischsprachigen Verbandes der Nutzer von Linux und von kostenloser Software (Association francophone des utilisateurs du Linux et des logiciels libres, AFUL) für die afrikanischen NRO, die sich für „freie Software“ einsetzen. Die Zusammenarbeit ist heute eines der Ziele des französischen Netzwerks der öffentlichen Zugangsstellen. Sie äussert sich durch die Bereitstellung seiner Kompetenzen auf dem Gebiet der Verwaltung und Leitung der Zugangsstellen, die Schulung (mit oder ohne Fernunterricht) und die Entwicklung von Dienstleistungen und Nutzungen für seine Kollegen des Südens ${ }^{18}$.

\section{Anwendungen in verschiedenen Bereichen}

Im Anfang betrafen die meisten Projekte internationaler Entwicklungszusammenarbeit den Infrastruktur- und Zugangsbereich. Sie waren bestrebt, auf dem Gebiet Afrikas Verbindungen, wie auch Computer- und Telematikausrüstungen zu entwickeln, die vor allem dem lokalen Kommunikationsbedarf der internationalen technischen Entwicklungshelfer sowie der Forscher und Akademiker (RIO und AUF) entsprachen. USAID subventionierte einen nationalen Knoten im Internet. Später wurde dieser Bereich im Verlauf der technologischen Weiterentwicklung der IKT im Westen und der Umsetzung der Politik zur Liberalisierung der historischen Telekommunikationsbetreiber dem privaten Sektor vorbehalten. Bis zum heutigen Tag im Jahr 2003 muss man feststellen, dass die reichen Länder - weit davon entfernt, sich für die Mitarbeit an Infrastrukturarbeiten einzusetzen, wie dies Nelson Mandela gewünscht hatte - die Entwicklungsländer vor allem dazu angetrieben haben, sich dem Weltmarkt der Telekommunikation und Dienstleistungen zu öffnen. Die Privatisierung der historischen Betreiber ist in rund zwanzig Ländern im Gang; die ausländischen Privatinvestoren haben sich die rentabelsten Marktlücken wie die Mobiltelefonie angeeignet und die leistungsfähigsten historischen Telekommunikationsbetreiber ihrer früheren Kolonien übernommen. Die Aktion der bilateralen amerikanischen Entwicklungszusammenarbeit USAID mit dem Ziel, den Anschluss dicht bevölkerter Städte zweiter Ordnung, wie jene der Demokratischen Repub-

18 Wir verweisen auf die Aktionen der Mitglieder des Verbandes Créatif (<www.creatif-public.net>), der seit Februar 2001 die Leiter der französischen öffentlichen Zugangsstellen zusammenschliesst; den Verband Mosaïque du monde (<www.mosaiquedumonde.org>), mit seinen Internet-Zugangsstellen in Schulen in den französischsprachigen Ländern, speziell im Süden; VECAM, mit seinen Initiativen für Partnerschaften zwischen französischen und afrikanischen Städten, Verbänden und Schulen (,i-jumelage“)(<www.villes-internet.net $>)$; Globenet (<www.globenet.org $>)$, usw. 
lik Kongo (Kananga, Mbuji-Mayi) durch Spenden eines halbjährigen InternetAbonnements an die lokalen NRO zu realisieren, ist originell.

Es gibt wenig begleitende Aktionen im Bereich der Reglementierung und Standardisierung der Kommunikationsprotokolle. Die Internationale Fernmeldeunion (ITU), die UNESCO, die UN-Wirtschaftskomission für Afrika (CEA) und das CRDI haben einige Programme zur Unterstützung bei der Festlegung eines Reglementierungsrahmens, der Internet-Nutzungen fördert, erstellt. Der afrikanische Gipfel über Internet und Telekommunikationen in Banjul (Gambia, 5.-9. Juni 2000) hat die Bedeutung dieses Bereichs hervorgehoben, indem er den Wunsch äusserte, dass sich in Afrika regionale Organisationen entwickeln, die fähig sind, nicht nur praktische Regulierungsprobleme betreffend die Entwicklung des Internets in Afrika zu lösen, sondern auch zu seriösen Gesprächspartnern der nationalen Verbände sowie auch der weltweiten Gruppen zur Regulierung und/oder Ausdehnung des Internets, wie die Internet Corporation for Assigned Names and Numbers (ICANN), die Internet Society (ISOC) usw. zu werden. Man kann heute folgende afrikanische Organisationen nennen, welche die Unterstützung der internationalen Zusammenarbeit erhalten haben: Africa Internet Registry (AIR), African Network Information Center (AFRINIC), African Network Operators Group (AFNOG), African Internet Service Providers Association (AFRISPA) ${ }^{19}$, usw.

Was die Internet-Zugänge betrifft, so bleibt die Option, gemeinschaftliche Internet-Cafés zu finanzieren, für mehrere Organisationen der Entwicklungszusammenarbeit noch insoweit prioritär, als die gemeinschaftlichen Internet-Zugänge im Allgemeinen für ein breites Publikum bestimmt sind, das in Bezug auf ein Einzelabonnement ,angeblich“ nicht sehr zahlungskräftig ist. Dies ist in der Tat die Hauptmöglichkeit, die in den letzten vier Jahren gewählt wurde, um die ländlichen Gebiete und die armen Bevölkerungsgruppen anzuschliessen (CRDI, Frankreich). Jedoch werfen die gemeinschaftlichen Internet-Zugänge das Problem ihres Fortbestehens nach dem Ende der ausländischen Finanzierungen auf, da die Erfahrungen vor Ort ergeben haben, dass sie gegenüber der Konkurrenz der privaten Unternehmen, welche dieselben Dienstleistungen zu oft gleichwertigen Kosten vertreiben, anfällig sind.

Im Gesundheitssektor wurde neben der Schwäche und der Unterfinanzierung der sanitären Infrastruktureinrichtungen ein schwerwiegender Mangel an Zugangsmöglichkeiten zu effizienter medizinischer Information, ein geringer Umlauf der Information über medizinische Forschung wie auch über die Behandlung und Verhütung von Krankheiten, die Überalterung der Infrastruktur im Bereich der medizinischen Information, das Verfallen der Bibliotheken sowie das fast völlige Fehlen von funktionierenden Telefonleitungen festgestellt. In seinem Weltbericht über die menschliche Entwicklung 1999 erwähnt

19 Die AFRISPA, deren Sekretariat sich auf der Insel Mauritius befindet, wurde anlässlich des African Computing and Telecommunication Summit gegründet, der vom 1. bis 2. August 2002 in Pretoria (Südafrika) stattfand. Er hat zum Ziel, über die Interessen der afrikanischen Internet-Zugangsanbieter zu wachen und die Internet-Anschlussmöglichkeiten in Afrika zu fördern. Er setzt sich ursprünglich aus den englischsprachigen afrikanischen Staaten, namentlich Südafrika, Ghana, Insel Mauritius, Kenia, Nigeria, Uganda und Tansania zusammen. 
das UNDP den typischen Fall des Kongos, wo die Universität Brazzaville lediglich über vierzig medizinische Werke und ein Dutzend Zeitschriften von vor 1993 verfügte, wie auch den Fall der Medizinischen Fakultät der Universität Nairobi, die nur noch zwanzig Zeitschriften (gegenüber 300 im Jahr 1997) erhielt. Der Beitrag der Entwicklungszusammenarbeit wird unter anderem im folgenden Prozess der Integration der IKT sichtbar: die Umsetzung der Initiative „Telegesundheit in Westafrika“" (Télésanté en Afrique de l'Ouest“), die seit 1999 in den Pilotgebieten von Saint-Louis und Diourbel dank der Partnerschaft zwischen der Universitätsklinik Dakar, der Acacia-Strategie des CRDI, der ITU und SONATEL funktioniert; die Schaffung medizinischer Online- und CDROM-Ressourcen; die Ausrüstung mit medizinischem Fernübertragungsmaterial; die Bildung von Verbänden ohne Erwerbszweck, welche den Gesundheitsbeauftragten frische Informationen zur Verfügung stellen (hierbei sei unter anderen das Forschungsnetz Network for Equity in Health in Southern Africa (EQUINET), die NRO Southern Africa AIDS Information Dissemination (SAFAIDS) oder das Handbuch der medizinischen Ressourcen University of Zambia Medical Library) erwähnt; und viele andere Initiativen, von denen einige Gegenstand von Informationsblättern über die Anwendung im Telemedizinbereich auf der Konferenz in Bamako $2000^{20}$ waren.

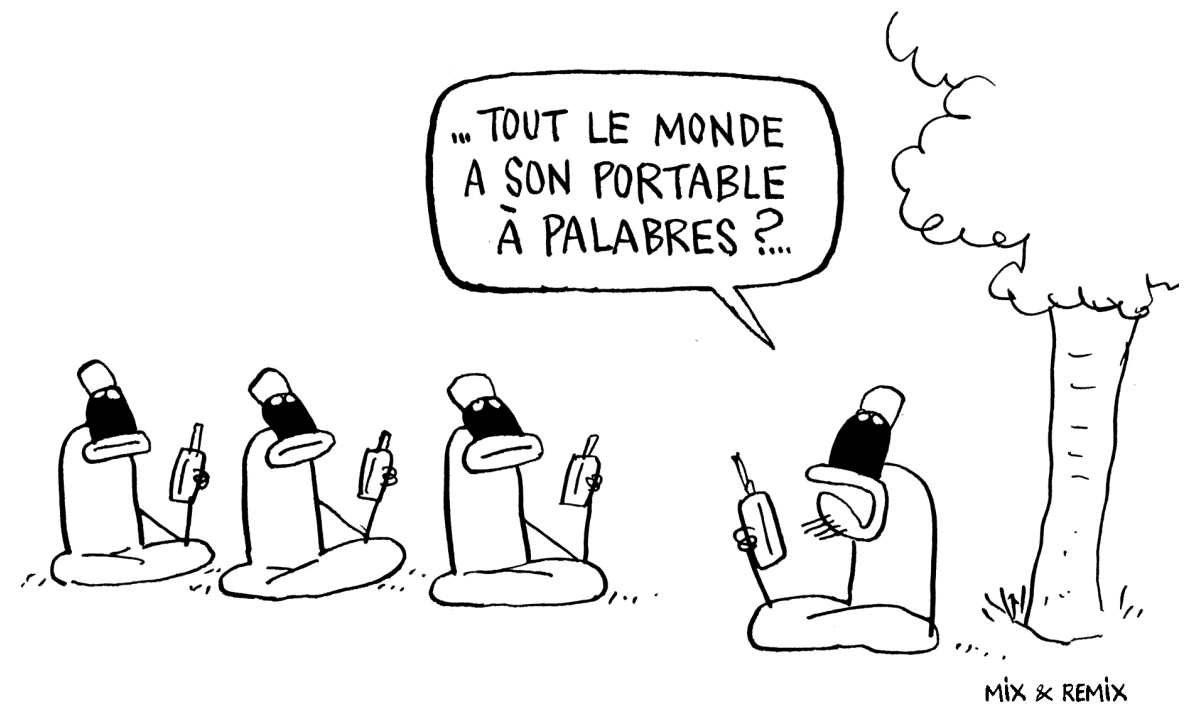

...Hat jeder das Palaver-Handy?...

20 Siehe : <www.anais.org >. 
Angesichts der Marginalisierung der afrikanischen Frauen gegenüber dem Informationsumlauf, des mangelnden Fachwissens der Frauen in den Bereichen Wissenschaft und Technik, der Nichtwahrnehmbarkeit der Tätigkeiten von Frauen auf nationaler, regionaler und internationaler Ebene, der festgestellten Ungleichheit der Geschlechter beim Zugang zu den IKT und ihrer Nutzung, kurz gesagt, angesichts der Ausgrenzung der afrikanischen Frau aus Gründen der Gesellschaftsorganisation in grossem Massstab setzt die Entwicklungszusammenarbeit sowohl Programme für den Anschluss und die Mobilisierung der Frauen bezüglich der Nutzung der IKT, als auch für den Zusammenschluss der Frauen in Verbänden zur Förderung ihrer Beteiligung an der Informationsgesellschaft $^{21}$, ein. Die NRO ENDA Tiers-monde erhält die Unterstützung des CRDI zur Verwirklichung der Ziele, die sie sich durch ihr Team Synergie genre et développement (SYNFEV) gesetzt hat, das sich seit 1995 dafür engagiert, die afrikanischen Frauen für eine verstärkte Nutzung der durch die elektronische Kommunikation gebotenen Möglichkeiten auszubilden. In diesem Zusammenhang sind auch die verschiedenen Workshops zur Einführung der Frauen in die IKT zu erwähnen. So zum Beispiel der Workshop vom 17. bis 21. Januar 2000, der Frauen aus den Staaten Benin, Burkina Faso, Elfenbeinküste, Mali, Mauretanien, Senegal und Togo zum Thema „Endogene Herstellung von Inhalten für Frauengruppen im französischsprachigen Afrika“" zusammenbrachte ${ }^{22}$. Die NRO Women Connect erhält 1999 von der Gates-Stiftung Subventionen von über einer Million US-Dollar, um den afrikanischen Frauen im ländlichen Bereich Anschlussmöglichkeiten zu bieten.

Was die ländlichen Gebiete betrifft, so war die Integration der IKT auf Strukturen ausgerichtet, welche die landwirtschaftlichen Tätigkeiten sichtbar machen und aufwerten, den Leitern im ländlichen Raum zur Wiederbelebung ihrer Arbeitswelt unerlässliche strategische Mittel und Kenntnisse zur Verfügung stellen, die traditionellen Aktivitäten diversifizieren sowie eine Wirtschaft der Dienstleistungen und der so genannten „neu aufkommenden“ Berufe einführen. Die Entwicklungszusammenarbeit hat dazu unter anderem Telezentren, Wissensdatenbanken für die afrikanische ländliche Bevölkerung, usw. beigetragen. In diesem Zusammenhang sei die Errichtung der Wissensdatenbank des Weltzentrums für landwirtschaftliche Informationen (WAICENT) erwähnt, die auf dem Web einsehbar ist und die Bereiche Landwirtschaft, Forstwesen, Fischerei, Nahrungssicherheit, nachhaltige Entwicklung sowie statistische Daten über Produktion und Handel mit landwirtschaftlichen Produkten umfasst. Ferner sei das Vorhaben von Inter-Réseaux erwähnt, ein Informationsregister über Orte, Organisationen und Methoden für das Sammeln, den Austausch und die Verbreitung strategischer Kenntnisse für die Akteure der ländlichen Entwicklung des Südens zu erstellen.

Die Schulung ist zur bevorzugten Option der Organisationen für Entwicklungszusammenarbeit geworden. Sie betrifft alle Ausbildungsniveaus, von den gewöhnlichen Nutzern über Techniker, Fachkräfte für Regulierung, Multimedia-

21 Zusätzliche Informationen zur Auswirkung der IKT auf die Genderfrage finden sich in M. Rathgeber, E. Ofwona Adera (dir.), L'inégalité des sexes et la révolution de l'information en Afrique, CRDI, 2000, 280 S., <www.idrc.ca/books/focus/941/01-front.html>.

22 Siehe: <www.enda.sn/synfev/synfev.htm>. 
Gestalter bis hin zu den Managern. Sie strebt die Stärkung der lokalen Kapazitäten an, die für eine nachhaltige Eingliederung der IKT in Afrika unerlässlich sind. Im Schulungsbereich zeichnen sich fünf Ausrichtungen ab:

$\checkmark$ Die Sensibilisierung für die Möglichkeiten des Internets. Dabei handelt es sich um Informationskampagnen, die in Form von Kolloquien, internationalen Zusammenkünften und sogar Demonstrationsveranstaltungen durchgeführt werden, wie das bei der "Internet-Karavane“ von OSIRIS im Senegal der Fall war. Die multilaterale Zusammenarbeit und die NRO waren in dieser Hinsicht sehr aktiv:

$\square$ Das Erlernen der digitalen Grundkenntnisse, das aus Kurztagungen zur Einführung in die Anwendung der üblichen Software wie Windows, Linux, Textverarbeitung, Tabellenrechner-Programme, Versand und Erhalt von EMails, Website-Editoren usw. besteht. Dies ist zweifellos der Bereich, in dem sich die Beteiligten aller Art, einschliesslich der speziell zu diesem Zweck gegründeten NRO, am meisten einsetzen. Das Programm Prosud, welches das Projekt Afriweb umfasst, mit dem Ziel, zwanzig WebsiteRedaktoren aus Benin, Burkina Faso, der Elfenbeinküste, Mali und dem Senegal auszubilden, ist ein Beispiel dafür. Die dezentralisierte und multilaterale Zusammenarbeit sensibilisiert für den Online-Anschluss lokaler Inhalte, indem sie die internationale Expertise in diese Herstellung - wiederum durch kurze Schulungstagungen für die Software-Anwendung - mit einbezieht. Einigen Websites mit kulturellem Inhalt, besonders jenen des nationalen kulturellen Erbes, kam dies zugute.

- Die klassische, vornehmlich berufliche, aber rasche Ausbildung der Verwalter der Telematiknetze. Trotz der legitimen Vorbehalte, die man zu dieser Art von Partnerschaft vorbringen $\mathrm{kann}^{23}$, ist es der Zusammenarbeit zwischen UNDP und Cisco gelungen, ein Programm zur Ausbildung junger Netzverwalter zu erstellen und dadurch zur Stärkung der „digitalen Kapazitäten“ in Afrika beizutragen. Die Cisco-Ausbildungszentren, sog. „Akademien“, die in mehreren afrikanischen Ländern eröffnet wurden, erteilen "Cisco“Diplome im IKT-Infrastrukturbereich, wodurch sie den wissenschaftlichen und technischen Disziplinen Vorrang einräumen. Ein Projekt der ITU sieht vor, bis Juli 2003 fünfzig Ausbildungszentren (davon $15 \mathrm{im} \mathrm{Jahr} \mathrm{2001,} 20 \mathrm{im}$ Jahr 2002 und 15 bis spätestens Mitte 2003) in Institutionen ohne Gewinnorientierung der Entwicklungsländer zu errichten, um dort die Technologie der Internet-Netzwerke und die diesbezüglichen Dienste zu lehren.

- Die Fernausbildung, die darauf abzielt, die Lücken des mangelhaften Bildungssystems in Afrika zu beheben und eine neue Form der Ausbildung mit Diplomabschluss auf Hochschul- und Universitätsniveau einzuführen. Multilaterale Organisationen wie die Weltbank und die Agence de la Francophonie richten ihre Aktionen auf die Gründung virtueller Universitäten aus. Die

23 Wir verweisen unter anderen auf die Artikel von P. Renaud, „Un partenariat qui suscite des interrogations. Point de vue sur la coopération CISCO-PNUD pour la formation d'Africains aux NTIC“, Auszug aus der Liste Africa'nti, 5. Juni 2001, unter <www.africanti.org> (ebenfalls veröffentlicht in Elie M. [dir.], Le fossé numérique. L'Internet, facteur de nouvelles inégalités ?, Problèmes politiques et sociaux, Nr. 861, 10. August 2001, S. 58-59) und von E. Bernard, „Académies Cisco en Afrique de l'Ouest: quel est le jeu des institutions internationales?“, Januar 2001, <www.accessit.org/bibliotheque/ dossiers/pnud_cisco.htm>. 
Agence de la Francophonie ist bestrebt, bei der Programmgestaltung die lokalen Lehrkräfte einzubeziehen. Das 1995 lancierte Weltbankprojekt mit der Bezeichnung „Afrikanische virtuelle Universität“ besteht darin, den Ländern südlich der Sahara Fernunterricht zu erteilen, um gegen den Wissensausschluss anzugehen und die Chancen für einen breiten Zugang zum Unterricht zu erhöhen. Dieses Projekt ist aus der Feststellung der Auslastung der Unterrichtsstrukturen und aus dem Defizit an Betreuungspersonal entstanden. Sein Ziel ist weiterhin, „den Hochschulunterricht zu fördern und die Kommunikationssatelliten einzusetzen, um Kosten und Schulgeld zu reduzieren“. Das unter dem Namen Global Development Learning Network, GDLN (Globales Netzwerk für Entwicklungsbildung) bekannte Projekt der Weltbank ist als Multimedia-Pilotzentrum für Fernausbildung konzipiert und soll mit Hilfe der Informations- und Telekommunikationstechnologien (Fernsprech-Bildkonferenzen, interaktiver Unterricht, Kommunikationssatelliten, Internet) die Fähigkeiten der afrikanischen Verantwortlichen und Entscheidungsträger des öffentlichen, privaten und Vereinssektors stärken. Die Zentren in Abidjan, Cotonou und Dakar sind afrikanische GDLN-Prototypen.

Die Weltbank hat das Programm Information for Development InfoDev (Information für die Entwicklung) geschaffen, das die IKT durch den Privatsektor und auch in diesem Sektor fördern soll, aber mehr Forschungsorgane oder öffentliche Instanzen der Länder des Nordens als des Südens finanziert. Die zentralisierende Konzeption der Weltbank drückt sich in ihrem Programm Global Knowledge Development (GKD) aus, das ein ,globales Wissen“ über die Entwicklung mittels eines Internet-Portals sammeln und zusammenfassen will ${ }^{24}$.

$\square$ Die klassische Ausbildung des formellen Bildungssystems, das die traditionelle Form der internationalen technischen Zusammenarbeit annimmt. Man kann hierzu das operationelle Programm des französischen Aussenministeriums mit dem Titel „Réseau d'appui francophone pour l'adaptation et le développement des TIC en éducation" (Netzwerk französischsprachiger Unterstützung zur Anpassung und Entwicklung der IKT im Bildungsbereich) anführen, das in Westafrika bereits über Zentren mit MultimediaRessourcen, Informationszugang, Ausbildungsräume und Räume für die Herstellung von Bildungsressourcen verfügt.

Die Stand-by-Netzwerke, welche die internationalen Organisationen einrichten wollen, sind zum Sammeln und Verbreiten von Informationen über ihre strategischen Bereiche bestimmt. Die Projekte zur Entwicklung der Web-Server, wie sie von der Banque internationale d'information sur les Etats francophones (BIEF) konzipiert sind, sollen zur aktiven Bereitstellung von zur Entscheidungsfällung nützlichen Daten dienen ${ }^{25}$.

24 Die Diskussion über die „Entwicklung des globalen Wissens“, die von der Weltbank gesponsert und nur in Englisch geführt wurde, befasste sich sechs Wochen lang mit der Frage, ob es zweckmässig sei oder nicht, ein Portal über die globale Entwicklung einzurichten. Siehe A. Chéneau-Loquay, Créer un portail global sur le développement?, Kurzinformation, <www.africanti.org/resultats/breves/gkd.htm>, und Bamako 2000. Notes sur le discours de $M^{m e}$ Aminata Traoré, April 2000, $<$ www.africanti.org/resultats/breves/bko-traore.htm>.

25 Siehe : <www.rfi.fr/Kiosque/SommetDeHannoi>. 
Im Jahr 2000 hat die UN-Wirtschaftskommission für Afrika mit der Unterstützung des kanadischen Forschungszentrums CRDI, der Europäischen Union und Norwegens die Initiative SCAN-ICT mit dem Ziel eingeleitet, „die Entwicklung der afrikanischen Kapazitäten zu fördern, um die Sammlung und Verwaltung der Schlüsselinformationen zu erfassen, welche die zunehmenden Investitionen in die Informationstechnologien sowie den Übergang Afrikas zur Informationsgesellschaft unterstützen sollen“. Dabei geht es um die Errichtung eines panafrikanischen Netzwerks über die Informations- und Kommunikationstechnologien, das alle Aspekte dieser Thematik umfasst und auf regionaler Ebene von einem wissenschaftlichen Ausschuss koordiniert und unterstützt werden soll. Der Ausschuss hätte die Rolle, die Arbeit auf nationaler Ebene zu erleichtern. Fünf Pilotländer - der Senegal, Ghana, Mosambik, Äthiopien und Marokko - wurden ausgewählt, um eine einjährige Pilotstudie durchzuführen, die zur Ausarbeitung einer Datenbank für jedes Land und zu einer Querschnittsanalyse führen soll.

Die internationale Begleitung bei der Formulierung der nationalen und regionalen IKT-Politik ist fortan ein wichtiges Anliegen der multilateralen Organisationen für Entwicklungszusammenarbeit. Zum Beispiel stehen das UNDP, die Wirtschaftskommission für Afrika (CEA), der Entwicklungshilfeausschuss der OECD und die Global Digital Opportunity Initiative (GDOI) bei der Ausarbeitung der Unterlagen für nationale Internet-Strategien immer mehr hinter den afrikanischen Ländern. Mosambik hat diesbezüglich Unterstützung von der GDOI erhalten, Nigeria und Tunesien von Italien, Benin und die Demokratische Republik Kongo vom UNDP, und auf regionaler Ebene wurde Afrika von der CEA über die African Information Society Initiative (AISI) unterstützt. Doch wenn die Unterlagen einmal ausgearbeitet sind, wie steht es dann mit der Umsetzung der Aktionspläne?

Man kann feststellen, dass nur wenige Programme das lokale Wirtschaftsgeflecht der privaten kleinen Unternehmen betreffen. Es ist unmöglich, den Finanzbetrag für die gesamte Entwicklungszusammenarbeit im IKT-Bereich zu kennen, jedoch ist er, trotz des vorherrschenden Eindrucks einer Vielfalt von Aktionen, nicht sehr hoch. Tatsächlich wird man sich vor Ort der relativ geringen Auswirkung der durchgeführten Projekte bewusst.

Wie fügt sich das Internet - so wie man es uns im Zusammenhang mit Afrika präsentiert und wie es sich exportiert - in die Logik der verschiedenen Organisationen für Entwicklungszusammenarbeit ein? Ist es Gegenstand klarer politischer Strategien zahlreicher afrikanischer Länder und gehört es zu dem, was das Wesen des gegenwärtigen politischen Kontexts ausmacht, nämlich Neugestaltung und Konsens? Das Internet wird im Allgemeinen als Instrument politischer Befreiung und als Mittel zur Verbesserung der Lebensbedingungen präsentiert. Entspricht es aber der lokalen Nachfrage oder eher der interventionistischen und „,programmatischen“ Politik der Geberländer des Nordens? Wird man die internationalen Spielregeln ändern, damit das Internet anhand seiner Finanzierung seine Rolle in Afrika voll und ganz spielen kann? 
All diese Programme der Entwicklungszusammenarbeit bezeugen die Tatsache, dass das Internet für die Akteure des Nordens zu einem echten Bedarf für die Entwicklung Afrikas geworden ist. Es handelt sich jedoch um einen noch „,virtuellen" Bedarf, das heisst von aussen her empfunden, als das was zu seiner Förderung im Vergleich mit dem Westen fehlt, und dessen bewusst zu sein, die Afrikaner lernen müssen. Mit anderen Worten geht es darum, nach der Projektion des Nordens auf das afrikanische Umfeld einen Mangel durch rasche Aneignung und gesellschaftliche Beherrschung auszugleichen. Das Internet erscheint hier als ein Bedarf, der zwar auch zu befriedigen, vor allem aber $z u$ definieren, vorzuschlagen und sogar durch verschiedene Aktionen der Entwicklungszusammenarbeit durchzusetzen ist. Während über das, was zu tun wäre und was vorgesehen ist, alles gesagt und geschrieben wurde, kennt man die tatsächlichen Auswirkungen der verschiedenen durchgeführten Aktionen schlecht. Die lokalen Beteiligten evaluieren ihre Aktionen nicht, die Auswirkungen sind unbekannt oder schwer messbar. Man spricht von positiven Erfahrungen, doch stellt man sich Fragen über die Möglichkeiten, sie allgemein anzuwenden ${ }^{26}$.

\section{Die Missionarsideologie}

Der allgegenwärtige Diskurs der internationalen Organisationen, welche die IKT zu ihrer neuen Priorität für die Entwicklung oder vielmehr, laut einem üblichen Begriff, für die „Armutsminderung“ gemacht haben, zeigt deutlich das Fortbestehen einer ,geistigen Hegemonie“ derjenigen, die wissen, - die Eliten des Nordens - gegenüber denjenigen, die lernen und nach für sie ausgearbeiteten Anweisungen handeln müssen. Da die Welt ins „Informationszeitalter“ eingetreten ist, geht es darum, zu gewährleisten, dass Politiken eingeführt werden, die allen auferlegt werden sollen. Der Diskurs der internationalen Organisationen $^{27}$ hat hier ein neues Mittel für den Ausdruck seiner Rhetorik gefunden ${ }^{28}$. Um nur den jüngsten Umschwung zu berücksichtigen ${ }^{29}$ : „Die neuen Technologien in den Dienst der menschlichen Entwicklung stellen" ist das Thema des Jahresberichts des UN-Entwicklungsprogramms (UNDP) von 2001. Der Bericht stellt fest, das die IKT ein noch nie dagewesenes Instrument sind, um der Armut

26 Siehe J.-M. Blanchard, „De la pertinence de l'usage de l'Internet dans les pays en voie de développement“", in J.-F. Soupizet, L. Gille (dir.), Nord et Sud numériques, Les Cahiers du numérique, Band 2, Nr. 3-4, 2001.

27 Der den Diskurs der westlichen Vertreter der Jahre 1980-1990 (Al Gore, Negroponte, Pierre Levy, De Rosnay) ablöst.

28 Siehe G. Rist (dir), Les mots du pouvoir. Sens et non-sens de la rhétorique internationale, Nouveaux Cahiers de l'iuéd, Nr.13, Genf, iuéd; Paris, PUF, 2003.

29 Im Fall Afrikas hat der Entwicklungsdiskurs dieser Organisationen mit dem Thema der IKT eine zweite Jugend gefunden; ihre neoklassische Ideologie des linearen Fortschritts greift dieses Thema auf, um die zögernden Staaten zu mehr Liberalismus anzuregen. Der Diskurs über die Revolution des 3. Jahrtausends folgt der Linie einiger medienbekannter Autoren und in erster Linie des Vizepräsidenten der Vereinigten Staaten, Al Gore, über eine globale Informationsinfrastruktur. 1995 nimmt die Weltbank einen lyrischen Ton an, um zur „Revolution der Information“ aufzufordern, „die Afrika eine dramatische Gelegenheit bietet, den Sprung in die Zukunft zu tun und mit Jahrzehnten der Stagnation und des Niedergangs zu brechen. Afrika muss diese Chance rasch ergreifen. Gelingt es den afrikanischen Ländern nicht in verstärktem Masse, aus der Revolution der Information Nutzen zu ziehen und auf der grossen Welle des technologischen Wandels zu surfen, so werden sie von ihr 
im 21. Jahrhundert ein Ende zu bereiten, da sie auf fast allen Ebenen der menschlichen Tätigkeit eingesetzt werden, praktisch überall und für alle genutzt werden können. Dies gilt auch, weil diese Technologien die Hindernisse der menschlichen Entwicklung beseitigen, indem sie sowohl die Wissenshemmnisse (da Arme wie Reiche dank Internet Zugang zur Information haben) als auch die Hindernisse betreffend die wirtschaftlichen Möglichkeiten beseitigen. Für Frau Sakido Fukuda-Parr, Direktorin des IKT-Programms im Rahmen des UNDP, steht Folgendes fest: Wenn auch die Langsamkeit der Produktivitätsgewinne und der Untergang der jungen Firmen (Start-ups) die Begeisterung dämpfen, den die New Economy in ihren Anfängen auslöste, so handelt es sich dabei um eine kurzfristige und engsichtige Überlegung, da der Wandel struktureller Art ist und sich nicht auf Börsenkurse und Wirtschaftswachstum beschränkt ${ }^{30}$. Somit ist die Sache abgemacht; man kann den technologischen Determinismus, der das Einheitsdenken prägt, nicht besser ausdrücken, als indem man schreibt, dass „der menschliche Fortschritt vom technischen Fortschritt abhängt“. „Auch in den Entwicklungsländern trägt die Erhöhung der Zahl der Nutzer zur Kostensenkung bei und die Technologien werden den lokalen Bedürfnissen angepasst; das Potenzial der IKT wird somit lediglich durch die Vorstellungskraft und den politischen Willen beschränkt".

Der gleiche Bericht stellt ferner fest, dass der „digitale Graben“ sich ausweitet, jedoch lassen sich die Experten nicht täuschen. „Zu glauben, dass es eine Zaubertechnologie gibt, um Analphabetismus, Krankheiten und Wirtschaftskrisen abzuhelfen, beweist, dass man nichts davon versteht, was Armut wirklich ist". Das grosse Wort ist gefallen, die Armut als Wurzel allen Übels, welche die Länder des Südens - und vor allem Afrika - kennzeichnet und umhüllt. Doch sei dem wie ihm sei, die Aufforderung, sich zu mobilisieren und die „Herausforderung" anzunehmen, bleibt bestehen, da sich die Akteure der lokalen Entwicklung sonst schuldig machen, beiseite zu stehen, und nicht von den Segnungen der Globalisierung profitieren werden. „Wenn die Akteure der Entwicklung bewusst die Explosion der technologischen Neuerung ignorieren (...), laufen sie Gefahr, sich zu marginalisieren. Dies liefe darauf hinaus, den armen Ländern günstige Gelegenheiten zu verweigern (...) ${ }^{\text {‘31 }}$.

Diese Art von Diskurs, der Vorschriften macht, der bestimmte Konzepte verwendet und andere wieder nicht („Herausforderung“, „Gelegenheit“, aber nicht „Ungleichheit“, „öffentlich“, „Ausbeutung“), der aus Aufforderungen, Ratschlägen ja sogar Drohungen besteht, ist sehr charakteristisch; es bleibt nur noch übrig, sich zu überzeugen und die Gebote des Präsidenten der Weltbank oder des Exekutivdirektors des UNDP als Verwahrer des universellen Moralbewusstseins zu befolgen, die eine feierliche Warnung an die internationale Gemein-

überrollt werden. In diesem Fall laufen sie Gefahr, in Zukunft noch stärker marginalisiert und wirtschaftlich stagnierend zu sein als heute“.

30 S. Fukuda-Parr, „Mettre les nouvelles technologies au service du développement humain“, présentation du rapport du PNUD 2001, in Haut Conseil de la coopération internationale, Biens publics mondiaux et coopération internationale. Nouvelle stratégie pour de nouveaux enjeux, Paris, Karthala, 2002, S. 96-107.

31 Marc Malloch Brown, Verwalter des UNDP, Vorwort des UNDP-Berichts Rapport mondial sur le développement humain 2001. Mettre les nouvelles technologies au service du développement humain. 
schaft richten ${ }^{32}$. Nie werden die Machtstrukturen in Frage gestellt, es ist immer die gleiche Rollenverteilung: den reichen Ländern obliegt es, über die Wirtschaftsfragen zu entscheiden; den Entwicklungsländern obliegt es, Massnahmen zu ergreifen, um gegen Armut, Korruption und schlechte Verwaltung vorzugehen.

Im gleichen Sinne interpretieren wir die von Guy-Olivier Segond anlässlich des Treffens Bamako 2000 gestellten folgenden Fragen: „Wie kann man der Bevölkerung des Südens helfen, sich der Entwicklung anzupassen? Wie kann man sie für die neuen Technologien sensibilisieren? Wie kann man ihr die nötigen Strukturen zur Verfügung stellen? Wie kann man es ihr ermöglichen, sich die Computer zu beschaffen, die für sie mehrere Jahre Gehalt ausmachen? Wie kann man ihr alle Feinheiten des Surfens und der E-Mail beibringen? Wie kann man die Software beherrschen? Wie Inhalte schaffen? Wie Reparaturnetzwerke heranziehen? Wie Nutzer ausbilden? Und last but not least, mit welchen finanziellen Mitteln?“.

Diese Art und Weise, den afrikanischen Internet-Bedarf für die Entwicklungszusammenarbeit darzustellen, gehört zu den Schemen der klassischen Entwicklungstheorien, bei denen man die Bedürfnisse der zu entwickelnden Gesellschaften entsprechend dem aufzuholenden Rückstand und in Bezug auf die entwickelten Gesellschaften definiert. Sie hat den Vorteil, sich direkt und harmonisch in den Aktionsbereich der Geldgeber und Dienstleistungssponsoren einzugliedern und diese zu veranlassen, die nötigen Mittel bereitzustellen, um den somit definierten Mangel auszugleichen. Sie erleichtert die Aufstellung des Budgets insofern, als die Tätigkeiten und Ressourcen sorgfältig im Voraus programmiert werden. Unter diesem Gesichtspunkt werden die Einführung und Aneignung des Internets in Afrika zu einem zu erfüllenden Auftrag, zu einem kurzfristig zu erreichenden Ziel; die Einheimischen erscheinen als Ausführende oder Nutzer, während die Planer, die Entscheidungsträger oder besser gesagt die Verantwortlichen des Projekts zur Einführung der neuen Technologien und zur Schulung für die IKT den Eindruck hinterlassen, die einzigen wirklichen Akteure zu sein.

\section{Einige Einblicke}

Die Situation ist von einem Land zum anderen sehr unterschiedlich, und wir ziehen es vor, statt zu versuchen, eine hypothetische Bilanz des im Bereich der Zusammenarbeit Erreichten zu ziehen, den Schwerpunkt auf einige für den afrikanischen Nutzer wesentliche Probleme zu legen, die jedoch von den westlichen Institutionen der Entwicklungszusammenarbeit nicht immer richtig wahrgenommen oder berücksichtigt werden.

\section{$\square$ Lokale Beteiligung}

Die in den 70er und 80er Jahren von den UN-Organisationen eingeleiteten Sensibilisierungskampagnen für die Computerisierung Afrikas hatten die lokalen

32 Gilbert Rist zeigt, wie man in diesen Diskursen die alten Themen wiederfindet, die den Heilsreligionen am Herzen liegen. Gilbert Rist, Le texte pris aux mots, op.cit., S. 25-41. 
Akteure schlecht und recht auf die Integration der IKT in ihre Entwicklungsprojekte vorbereitet. Anschliessend versuchten die afrikanischen Länder nach und nach, ihr Problem der Entwicklung des Computerparks, der Heranbildung des lokalen Fachwissens und der Verbesserung der nationalen EDV-Politiken entweder selbst oder mit der Unterstützung der internationalen technischen Zusammenarbeit zu lösen. Um uns aber auf die Periode der Entwicklung des Internets Ende der 80er Jahre zu beschränken, sind drei Arten lokaler Beteiligung hervorzuheben: die passive Beteiligung, die Formulierung eines auf regionaler Ebene anwendbaren Standpunkts und die Mitwirkung an der globalen Aktion der internationalen Gemeinschaft.

\section{Die Beteiligung in Form von Ablösung}

Die erste Beteiligungsform ist für die Zeit von Ende 1980 bis gegen 1994 kennzeichnend. Sie widerspiegelt die Konzeption einer internationalen Zusammenarbeit, die auf die Projekte zur Öffnung nach aussen der afrikanischen Forschungszentren und der internationalen Entwicklungsorganisationen beschränkt ist. Die lokalen Akteure sind einfache Ausführende der Projekte, die sie nur insoweit betreffen, als sie Hilfskräfte oder „Partner“ der externen Geldgeber oder von deren Empfängern sind. Sie kommen im Allgemeinen aus den Kreisen der Wissenschaft. Sie lassen die Projekte zur Entwicklung der Netzwerke, die von den ausländischen Forschungsorganen errichtet werden, über sich ergehen und versuchen, sich ihnen anzupassen. Es wird ihnen jedoch gelingen, sich dank der technischen Ausbildung, die für die lokalen Verantwortlichen der Universitätsknoten bestimmt ist, als Ablösung heranzubilden, indem sie Aufgaben übernehmen, die einstmals für ihre Partner des Nordens vorgesehen waren. So werden die nationalen Domain-Namen (.sn, .ml, .bf, usw.), die in Westafrika in Partnerschaft mit RIO geschaffen wurden und von Montpellier aus verwaltet werden, von den lokalen Forschungszentren und Schulen bewirtschaftet.

\section{Die Formulierung eines afrikanischen Standpunkts zu den IKT und zur Zusammenarbeit}

Nach 1992 und mehr noch nach 1994 versuchen einige afrikanische Staatsführer, zu einer eigenen Vision der IKT zu gelangen. Sie engagieren sich sowohl in einer Kampagne zur Sensibilisierung ihrer Staatsbürger für die Priorität der IKT-Thematik, als auch in der Einführung des Internets. Anlässlich der Eröffnungsfeier der Telekom 1995 bei der ITU in Genf integriert Nelson Mandela die Telekommunikationsproblematik in einen politischen Rahmen globaler Entwicklung, der von den Gesetzen des Marktes befreit ist und somit über den alleinigen Handelssektor hinausgeht. Er drückt den Wunsch aus, der Telekommunikationsbereich möge zum Anliegen der Entwicklungszusammenarbeit werden. Im Oktober 1996 in Genf befürwortet Alpha Oumar Konaré, zu jener Zeit Präsident von Mali, eine Partnerschaft im Bereich der Informations- und Kommunikationstechnologien, die auf der afrikanischen Anerkennung der sozioökonomischen Bedeutung dieser Technologien und auf einem Internet beruht, das den von den Afrikanern vorher vorbereitetem afrikanischen Inhalt befördern sollte. Die regionalen Zusammenkünfte über IKT und Entwicklung, namentlich die Tagung der UN-Wirtschaftskommission für Afrika (ECA), welche die Initiative der afrikanischen Informationsgesellschaft (African Information Society 
Initiative, AISI) hervorbrachte, die Konferenzen von Bamaco 2000 und 2002 sowie die Treffen von Afrinet 1999 und 2000 betonen, obwohl sie von aussen finanziert wurden, die afrikanische Besonderheit als Zugangsweg zur Informationsgesellschaft und konzentrieren sich auf Aktionen zur Aneignung der IKT. Diese Aktionen sind unter anderem die Entwicklung von Infrastruktureinrichtungen auf dem gesamten Kontinent, die Verbindung über die Landesgrenzen hinaus, die Einbeziehung der traditionellen Telekommunikationsbetreiber bei der Erhöhung des Durchlassbereichs, eine Anreizpolitik zugunsten von IKTAusrüstungen und die Errichtung der Reglementierungsrahmen. Von nun an gilt Folgendes: Auch wenn man auf die lokalen Standpunkte verzichten kann, so weiss man, dass sie existieren und jederzeit ein Hindernis für unilaterale Initiativen bilden können.

\section{Die lokale Einbeziehung in die globale Aktion der internationalen Gemeinschaft}

Wenn auch mehrere Entwicklungsländer bis 1999 immer noch keine nationale gesamtheitliche Politik im IKT-Bereich eingeführt hatten, waren sie dennoch fast alle unter dem Druck der internationalen Gemeinschaft engagiert, Reformen des Telekommunikationssektors zu formulieren, die auf der Liberalisierung der öffentlichen Betreiber, dem Wettbewerb, den Privatkapitaleinlagen und somit auf der Marktöffnung basierten. Die Internet- und Mobiltelefoniebereiche waren von der Privatisierung am meisten betroffen. Der Senegal, Südafrika, Uganda und viele andere Länder haben aus dieser Politik zur Ausbreitung der IKT auf ihrem Staatsgebiet einen - wenn auch beschränkten - Nutzen gezogen. Auch haben die nationalen EDV-Pläne, welche man Anfang der 90er Jahre in mehreren französischsprachigen Ländern Afrikas antrifft, zu einer raschen Erweiterung des Computerparks beigetragen. In Burkina Faso beispielsweise konnte der Computerpark durch den Fünfjahresplan im EDV-Bereich von 1000 Geräten im Jahr 1990 auf 2700 im Jahr 1995 verdreifacht werden. Mit der dem Internet eingeräumten Priorität und der hohen Internet-Nachfrage in vielen afrikanischen Ländern werden mehrere lokale Firmen teils als Internet-Zugangsanbieter, teils als Dienstleistungsanbieter für die Entwicklung und Unterbringung der Websites gegründet ${ }^{33}$.

$\mathrm{Ab}$ dem Jahr 2000 ergreifen die lokalen Akteure immer mehr Initiativen, um sich an der weltweiten Bewirtschaftung der IKT zu beteiligen und diese in verschiedene Entwicklungsprojekte $\mathrm{zu}$ integrieren. Sie nützen das für die Zusammenarbeit im IKT-Bereich günstige internationale Klima aus und bedienen sich diverser in den 90er Jahren eingerichteter Strukturen, um neue Aktionen einzuleiten. Mehrere Vereinigungen entstehen, die an der Förderung der IKT arbeiten. Einige nehmen Projekte der Entwicklungszusammenarbeit nach der Art der gemeinschaftlichen Internet-Cafés auf, andere führen lokale Schulungen über das Internet ein, wieder andere setzen sich für die Einführung einer afrikanischen Informationsgesellschaft ein, usw. Regierungen setzen ihre Bemühungen zur Formulierung nationaler IKT-Strategien fort. So haben beispiels-

33 Eine Liste der in Afrika zu jener Zeit bestehenden Internet-Zugangsanbieter findet sich in B. Humbaire, „L'Internet et l'Afrique. Ressources documentaires“, Afrique contemporaine, Nr.182, 2. Quartal 1997. Siehe auch die von M. Jensen aktualisierte Website African Internet Connectivity, <http://demiurge.wn.apc.org:80/africa>. 
weise die Demokratische Republik Kongo und Benin ihre Projekte im April bzw. im Juli 2002 der internationalen Gemeinschaft unterbreitet. Universitäten wie jene in Kenia und Südafrika bieten Schulungen über das Linux-Programm an, usw.

Zur selben Zeit traten afrikanische Staatsoberhäupter in Erscheinung, um mehr Hilfe zu beantragen und eine neue Partnerschaft zu definieren. Dabei handelte es sich um das „Programm zur Wiedergeburt Afrikas“ des südafrikanischen Präsidenten Tabo Mbeki anlässlich des Weltwirtschaftsforums in Davos und um den „Omega-Plan für Afrika“ des senegalesischen Präsidenten Abdoulaye Wade $^{34}$. Diese Pläne führten zu einer gemeinsamen Initiative von vier Staatschefs unter dem Namen "Neue Partnerschaft für die Entwicklung Afrikas“ (NEPAD). Die Initiative tendiert dazu, für die Geldgeber bei der Festlegung von Politiken der Entwicklungszusammenarbeit, welche den Versuch Afrikas berïcksichtigen, seine Entwicklung selbst zu gestalten, zur Referenz zu werden. Der Teil „Neue Informationstechnologien“ stärkt die Idee eines afrikanischen Standpunkts in diesem Bereich. Desgleichen wird das Bestehen der NEPAD zum Garanten der IKT-Fragen auf Regionalebene.

All diese lokalen Initiativen entsprechen der immer weiter steigenden Nachfrage, die aufgrund der Erkenntnis der durch die IKT gebotenen Möglichkeiten für alle und für verschiedene Tätigkeitsbereiche entsteht. Jedoch hat man heute den Eindruck, dass diese Initiativen den von der internationalen Entwicklungszusammenarbeit vorgegebenen (privaten) Weg einschlagen und Gefahr laufen, mit der „Hilfemüdigkeit“ und dem Ende der „Pilotprojekte“ an Dynamik zu verlieren.

\section{$\square$ Weiterhin bestehende Infrastruktur-, Zugangs- und Ausrüstungsprobleme}

Man neigt in den reichen Ländern dazu, die Sachzwänge zu übersehen, denen die Afrikaner - auch die relativ gut ausgestatteten - im täglichen Leben ausgesetzt sind. Unzulänglichkeit und Qualität des Zugangs bleiben weiterhin ein wesentliches Problem, und es gibt immer noch keine grosse subventionierte Telekommunikationsausrüstungsprogramme, um den Zugang zum einfachen Telefon für die breite Masse zu ermöglichen. Die ITU scheint sich damit zu beschäftigen; doch wurde das Projekt zur Entwicklung afrikanischer Satelliten (Regional African Satellite Communications Organisation, RASCOM), das den Anschluss der ländlichen Gebiete ermöglichen würde, noch nicht gestartet, und es gibt nur wenige Länder, deren Gebiet mit einem wirklichen Maschennetz an Telekommunikationsinfrastrukturen, aber auch an ergänzenden terrestrischen Kommunikationsnetzen überzogen ist.

Beim Aufbau der vor der Einführung des Internets bestehenden Netze hat die Entwicklungszusammenarbeit eine grundlegende Rolle gespielt, doch sollte der Privatsektor anschliessend an die Stelle treten. Die ausländischen Privatinvesto-

34 Der „Omega-Plan“ wurde vom senegalesischen Staatspräsidenten A. Wade im Januar 2001 auf dem Frankreich-Afrika-Gipfel in Jaunde präsentiert. Sein Ziel ist, die grundlegenden Strukturunterschiede zwischen den entwickelten Ländern und den Entwicklungsländern durch auf Kontinentsebene koordinierte externe Investitionen abzubauen, um die Entwicklung in Afrika einzuleiten. Die betroffenen Bereiche sind insbesondere Infrastruktur, Gesundheitswesen, Bildung und Landwirtschaft. 
ren eigneten sich die rentabelsten Bereiche, wie die Mobiltelefonie, und einige von ihnen die Bereitstellung des Internet-Zugangs an, jedoch haben sich nur wenige dazu entschlossen, die schlecht gestellten historischen Betreiber zu übernehmen. Die ärmsten Länder sind gegenüber dem Ausbleiben von Aufkäufern machtlos und fragen sich, - wie Madagaskar - was sie in diesen Stadium tun sollen. Die Dienstleistungsfirmen zögern, wegen der hohen Kosten im Verhältnis zur Kundenzahl, sich ausserhalb der wichtigsten Städte auszubreiten. Eine Politik der Unterstützung für ihre Niederlassung auf der Grundlage öffentlich-privater Partnerschaftsformen wird von einigen Ländern wie Mali empfohlen.

Die durch den Staat angetriebene Entwicklung der Netzwerke in Europa bildete die Grundlage der industriellen Entwicklung. Es wurden Ausgleichspolitiken eingeführt, um dem Benutzer von der Gebietslage unabhängige Preise anzubieten, gleich ob er in der Stadt oder in einem schwer zugänglichen Randgebiet lebt. In Frankreich erhalten die Unternehmen staatliche Beihilfen, um sich in benachteiligten Gebieten niederzulassen. Diese Art von Lösungen scheinen für die afrikanischen Länder nicht zu existieren, wo ,alles viel teurer ist als anderswo“, wobei dies stets mit der Beschränktheit des zahlungsfähigen Marktes begründet wird, während nachgewiesen wurde, dass das Telefon im ländlichen Raum zum Beispiel ein rentables Geschäft sein kann, und dass das Mobiltelefon einen Aufschwung verzeichnet hat, der auch die grössten Skeptiker erstaunte. Die wenigen Projekte von NRO, die ein Dorf mit einem Telefon ausstatten, geben zwar den Betreffenden momentane Befriedigung, doch sind sie nur Linderungsmittel und können keine Politik einer abgestimmten Entwicklung der Netze sowie eine Raumplanung ersetzen, die in erster Linie dem Staat obliegt.

Die Frage der in Afrika subventionierten öffentlichen Gemeinschaftszugänge wirft ein besonderes Problem auf, das in Europa nicht besteht. In dem Masse, als die Hauptform des Internet-Zugangs in Afrika aus Kostengründen kollektiv und nicht individuell ist, üben die gemeinschaftlichen Telezentren - die niedrigere Preise praktizieren können als die anderen, von privaten Betreibern bewirtschafteten Zentren - einen unlauteren Wettbewerb aus. Nun lässt aber der Telefon- und Internet-Zugang ein Geflecht kleiner Privatunternehmen entstehen, die zahlreiche Arbeitsplätze in den Städten schaffen. Diese Gemeinschaftszentren haben eine Pionierrolle bei der Verbreitung der Nutzung des Web gespielt. Sie sollten aber von jetzt an eher in den benachteiligten Gebieten - Armenviertel, Kleinstädte - eingerichtet werden und zur Schulung der anderen Betreiber dienen, Vereinigungen mit genau festgelegten Zielen gehören, oder in öffentlichen Schulen installiert werden.

\section{$\square$ Eine minimalistische Konzeption}

Wenn einige Länder die Einfuhrabgaben auf Computergeräte gesenkt haben, so stellt dies - da wo das ungenügende Steueraufkommen bereits ein Grundproblem bildet - einen Verdienstausfall für die nationale Gemeinschaft dar. Das Fehlen von Computerindustrien und die geringe Anzahl von Computer-Montagebändern tragen nicht zur erforderlichen technischen Beherrschung bei, ohne die eine vollständige Abhängigkeit besteht. Die Bereitstellung von Gebrauchtcomputern an gemeinschaftliche Internet-Zentren oder an lokale NRO durch Körperschaften des Nordens ist eine sich verbreitende Praxis, die aber ange- 
fochten wird. Die rasche Weiterentwicklung der Werkzeuge verkürzt die Zeit ihrer normalen Abschreibung und grenzt praktisch an Verschwendung. Dies ist insofern der Fall, als man eine Technologie aufgibt, bevor man sie genügend ausgewertet hat. Durch das Sammeln alter Computersysteme in entwickelten Ländern macht man das Material zu einem Gegenstand der Zusammenarbeit mit dem Süden. In dieser Perspektive sammelt die amerikanische NRO World Computer Exchange ${ }^{35}$, mit Sitz in Massachusetts, veraltetes Computermaterial in den USA (das ans Internet im Süden angeschlossen werden kann), um es „kostenlos" an Schulen und Gemeinschaftszentren in den Entwicklungsländern (Afrika, Asien und Lateinamerika) zu liefern. Wie man sieht, dient die Entwicklungszusammenarbeit zwar dem lokalen Bedarf, spielt aber auch eine Rolle bei der Sanierung der durch den westlichen technologischen Entwicklungsrhythmus verursachten Situationen und bei der Wiederverwertung der Computergeräte, eine einfache Art und Weise, sich toxischer Produkte zu entledigen ${ }^{36}$.

Somit bildet sich eine minimalistische Konzeption der IKT heraus, die den westlichen Gesellschaften die Legitimität einräumt, in den Genuss aller Neuerungen zu kommen, und Afrika die Wahl der angeblich ,angepassten“ Technologien lässt, - welche im Allgemeinen veraltet sind, keine Aussichten auf lokale wissenschaftliche Verbesserung haben und von der alltäglichen „Findigkeit" der Betroffenen abhängen, - unter dem Vorwand, dass die neuesten Anwendungen nicht unbedingt mit dem Bedarf und den Mitteln der afrikanischen Gesellschaft übereinstimmen ${ }^{37}$. Dieser IKT-Ansatz, den viele als pragmatisch betrachten, richtet die Entwicklungszusammenarbeit auf minimalistische Initiativen aus, die der Armut und nicht dem Kampf gegen die Armut angepasst sind. Anstatt eine Dynamik der Bedürfnisse zu erzeugen, die alle Dimensionen der IKT verständlich macht, trägt diese restriktive Konzeption eher dazu bei, den afrikanischen Status quo anzuerkennen, und überlässt der internationalen Zusammenarbeit die Initiative, die Erschliessung sämtlicher Dimensionen der IKT zu beschleunigen, oder auch nicht.

$35<w w w . w o r l d c o m p u t e r e x c h a n g e . o r g>$.

36 Die Computerabfälle (E-waste) werden in den nächsten Jahren stark zunehmen. Man schätzt, dass es im Jahr 2005 einen veralteten Computer für jeden auf den Markt gebrachten neuen Computer geben wird. Bereits heute werden allein im US-Bundesstaat Kalifornien täglich 6000 Computer ausgemustert. Für 2001 macht das allein für die Vereinigten Staaten 41 Millionen Computer zum Wegwerfen aus. Nun enthalten die Computerabfälle aber an die tausend verschiedene Substanzen, von denen viele giftig sind (Blei, Quecksilber, Kadmium, Beryllium, Arsen, usw.). Ein Teil dieser Abfälle wird in den Ländern, die sie herstellen, vor Ort verbrannt; ein ganz geringer Teil wird wiederverwertet, ein weiterer Teil (80\% gemäss den Quellen) wird in Entwicklungsländer ausgeführt, wo die Umweltgesetze viel lockerer sind. Vgl. E. Bernard, Techno-trash en Asie, Kurzinformation, <www.africanti. org/resultats/breves/techno-trash.htm>.

37 Es werden Reden laut, wie: „Die Abgenutztheit eines Modems hat wenig Bedeutung, die Hauptsache ist, dass es uns anschliesst“; „Das ist besser als gar nichts“; „Wozu braucht es Pentium-4-Prozessoren für ein Afrika der E-Mail ?“, usw. Andere Gesprächspartner gehen sogar soweit, die Weiterentwicklung der Technologie zu leugnen, indem sie unter anderem argumentieren, dass „,es keinen Unterschied zwischen einem Pentium 3 und einem Pentium 4 gibt“, ,,dass das Windows XP den afrikanischen Benutzern, die lediglich 5\% seiner Kapazitäten anwenden, nichts Neues bringt“", usw., als ob sich die Nutzung der IKT auf die von den Internet-Cafés angebotenen Dienstleistungen oder auf den unmittelbaren Bedarf einiger Benutzer beschränken würde. 


\section{$\square$ Eine Konzentration auf die Ärmsten}

Im gleichen Sinne entspricht die Tatsache, die Aktionen der Entwicklungszusammenarbeit im Namen der „Armutsbekämpfung“ prioritär auf die ärmsten Bevölkerungsgruppen auszurichten, vielleicht nicht dem „,besten Vorgehen“ zur Verbreitung des Internets, da diese den Einsatz einer Technologie (Computer und dazugehörige Software) erfordert, welche komplex, kostspielig und von sehr ungleich verteilten Basis-Infrastruktureinrichtungen (Telefon und elektrische Energie) abhängig ist, und auch deshalb, weil das Internet den Lese- und Schreibkundigen vorbehalten ist. Das Programm Acacia empfiehlt seit 2001 - nachdem es festgestellt hatte, dass sich die Abstände zwischen dem Norden und dem Süden vergrössert haben, und indem es gleichzeitig weiterhin die soziale Investition für die Anwendungen der IKT fördert - auch Nachforschungen in Richtung auf den aussichtsreichsten Markt (front of the market) ${ }^{38}$. Unsere Erhebungen im Senegal zeigen insbesondere, dass eine Nachfrage nach finanzieller Unterstützung und Ausbildung seitens der privaten kleinen Unternehmen besteht, die das Wirtschaftsgeflecht bilden (Internet-Zentren, Computermontage und -reparatur, usw.). Jedoch berücksichtigen die Instanzen der Entwicklungszusammenarbeit selten den afrikanischen Privatsektor ${ }^{39}$. Zum anderen sollte sich das Streben nach Konvergenz zwischen EDV-Technik, Audio-Video-Technik und Telekommunikationen, das bis dahin vernachlässigt wurde, besonders bei den Ausrüstungsprogrammen der ländlichen Kommunen entwickeln ${ }^{40}$. Indessen hat das Internet Tendenz, allein im Rampenlicht zu stehen, während das wichtigste Informations- und Kommunikationsmittel in Afrika weiterhin das Radio - als einziges auf einer gesamten Gebietsfläche zugängliches Mittel - bleibt. Der nationale Rundfunk und die lokalen Radiosender spielen eine wesentliche Rolle als bevölkerungsnahe bzw. interaktive Medien. Wenn die Radios mit der Zeitschriftenpresse assoziiert sind, (wie in der Gruppe Süd im Senegal) und eine Internet-Verbindung haben, werden sie zu sehr wirksamen Instrumenten der Informationsverbreitung und sogar der Bildung ${ }^{41}$. Die Agenturen tendierten dazu, sich auf eines der Medien zu spezialisieren, doch scheint sich die Praxis zu ändern. Panos, in den herkömmlichen Medien (Rundfunk und Presse) spezialisiert, beginnt sich für deren Verbindung mit Internet zu interessieren.

\section{$\square$ Die Förderung der freien Software}

Einige Analytiker sind der Ansicht, dass der Rhythmus der Software-Entwicklung für die IKT durch das System der Software-Patente, der stillgelegten Lizenzen und des Verkaufs von Eigentümer-Software verlangsamt wird. Dies geht aus den Forderungen der sozialen Bewegung hervor, die für die Idee eintritt, der gesamten Gemeinschaft kostenlose Software zur Verfügung zu stellen. Das bedeu tet EDV-Programme, deren Quellcode für alle zugänglich, uneingeschränkt veränderlich und mit späteren Änderungen kostenlos oder zu viel geringeren Kosten

38 Siehe Acacia, Prospectus 2001-2005, Abschnitt „Lessons Learned“, <www.idrc.ca/acacia/prospectus 01_05.html\#lessons>.

39 Während die indische Erfahrung mit dem Mikrokreditwesen beispielsweise durchaus überzeugend ist.

40 Vgl. zum Beispiel das Programm Borgounet in Benin : <www.borgou.net>.

${ }^{41}$ Cf. Chéneau-Loquay A., ,L'Etat, le droit et les réseaux techniques dans le processus de modernisation en Afrique“, Terminal, Nr. 84, 2001, S. 55-82 <www.africanti.org/resultats/documents/ ACLTerminal1.PDF>. 
als die Eigentümer-Software vom Typ Microsoft verfügbar sind. Diese Konzeption rechtfertigt eine Zusammenarbeit mit dem Süden, die gleichzeitig auf die gemeinsame Ethik der „freien Software“ und ihrer Verbreitung ausgerichtet ist. Sie engagiert die Partner in einer Form der Zusammenarbeit, die den Schwerpunkt auf die Kreativität und die lokale Software-Innovation legt und somit der Schulung und dem Wissensaustausch Priorität einräumt. Die Initiativen von AFUL (Association Francophone des Utilisateurs de Linux et des Logiciels Libres) in den afrikanischen Ländern südlich der Sahara sind bis heute, Anfang 2003, Beispiele dieser Konzeption. Leider werden sie oft auf halber Strecke eingestellt, insofern als sie die Ausbildung vernachlässigen, welche die Softwareproduktion erfordert, und dann mehr der Ideologie, als der eigentlichen technologischen Innovation dienen. Andere Initiativen in Partnerschaftsform zwischen Forschungs- und Ingenieurausbildungszentren - Nord-Süd (CREIS ${ }^{42}$-CNRS) und Süd-Süd (Technische Fachschule Dakar) - ermöglichen eine aktivere Beteiligung an der Innovation und ihrer Verbreitung. In allen Fällen müssen die Lösungen der freien Software, um dauerhaft und gänzlich vorteilhaft zu bleiben, einer globaleren Perspektive der Errichtung lokaler Software-Industrien folgen, welche die unrentablen Abhängigkeiten verringern sollen.

\section{$\square$ Die Frage der Dauerhaftigkeit}

Die Analyse der Projekte der Entwicklungszusammenarbeit mit Afrika südlich der Sahara im Internet-Bereich hinterlässt oft den Eindruck, dass das Heil Afrikas in der Nutzung der IKT liegt, und dass es genügt, in die Erlernung der Software-Instrumente, das Heraufladen des afrikanischen Inhalts auf das Web und die Telematik-Infrastruktur zu investieren, um den Teufelskreis der gegenwärtigen Unterentwicklung zu durchbrechen. Die Reden an die Afrikaner geben mehr Empfehlungen ab, als dass sie sich auf die konkrete Art und Weise beziehen, wie die Firmen des Nordens die Schwierigkeiten gelöst haben und weiterhin lösen, die mit der Neuheit der IKT und ihrer Einführung in die öffentlichen Verwaltungen, Unternehmen und gesellschaftlichen Bräuche verbunden sind. Man begreift dann, warum man auf den Konditionalitäten besteht und warum man - wenn es um Afrika geht - die Hindernisse und die zur Einführung der afrikanischen Informationsgesellschaft fehlgeschlagenen Initiativen dramatisiert.

Nehmen wir ein Beispiel ${ }^{43}$. Gewisse Informationen, die aus den Organisationen $\mathrm{zu}$ uns gelangen, welche versuchen, die IKT in ihre laufende Betriebsführung zu integrieren, ergeben unter anderem, dass die IKT heute in den afrikanischen Unternehmen und NRO insoweit ein Faktor des Rückschritts sein können, als sie das bisherige Kräfteverhältnis verändern. Wenn das Internet nur von den Vorgesetzten oder ihren vertrauten Untergebenen benutzt wird, steigen diejenigen, die es zu nutzen verstehen, leicht in der internen Rangordnung auf oder erhalten mehr Aufmerksamkeit seitens der Vorgesetzten, was das verborgene, aber echte Desinteresse einer Mehrheit der Beschäftigten, die Demotivation einiger Angestellten und somit Funktionsstörungen zur Folge hat.

42 Centre de coordination pour la recherche et l'enseignement en informatique et société.

43 Weitere interessante Beispiele findet man in dem Buch von J.P. Brunet, O. Tiemtoré, M.-C. Vettraino-Soulard, Les enjeux éthiques d'Internet en Afrique de l'Ouest. Vers un modèle éthique d'intégration, Paris, L'Harmattan, 2002, 186 S. 
Die IKT können in den Unternehmen in Afrika eher die Ausgaben erhöhen, als Gewinne einbringen: „Seitdem es das Intranet in unserer Abteilung gibt“, bezeugte ein Buchhalter, ,steigen die Kommunikationsaufwendungen. Die Angestellten verbringen mehr Zeit damit, zu surfen, als für die Firma zu arbeiten, die privaten E-Mails sind zahlreicher als die dienstlichen Mails. Die Neugier der Angestellten ist für unser Unternehmen zu einer Ausgabe geworden“.

Dieser Tatbestand ist nicht auf Afrika beschränkt. Vergleichende Studien könnten erklären, wie einige Unternehmen vorgegangen sind, damit die Nutzer der IKT im Unternehmen nicht nur für die Anwendung, sondern auch für die Einsätze dieser neuen Technologien im Management, in der Unternehmenskultur und in den menschlichen Beziehungen geschult werden. Man könnte Beispiele von Unternehmen anführen, welche die IKT banalisiert haben, nicht indem sie diese in den Hintergrund drängten, sondern indem sie sie zu Werkzeugen wie die anderen machten, nicht mehr im Dienst der persönlichen Macht oder der direkten Benutzer, sondern des gesamten Unternehmens.

Was die Beständigkeit der IKT-Projekte in Afrika betrifft, so wird heutzutage ein wirkliches Unbehagen dabei empfunden, Projekte zu verwalten, deren Lebensfähigkeit über ihre externe Finanzierung hinaus nicht gesichert ist. Die Versuchung wird sogar sehr stark, nichtstaatliche Organisationen in rentable Unternehmen umzuwandeln oder gewisse Aktionen der Vereinigungen ohne Erwerbszweck in gewinnbringende kommerzielle Tätigkeiten zu verwandeln. Es kann eine Psychose bei der Vorstellung entstehen, dass die IKT-Projekte, in denen man sich engagiert, wie so viele andere Projekte ohne Auswirkung - ausser der Verantwortung für den Fehlschlag und die „afrikanische“ Sünde der Verschwendung, Unfähigkeit oder Korruption - untergehen werden.

Die Begleitumstände der Fehlschläge der IKT-Pojekte sollten es gestatten, folgende Tatsachen zu berücksichtigen:

- Das Dauerhaftigkeitskonzept könnte im afrikanischen Kontext neu definiert werden, indem man einem Projekt die Fähigkeit einräumt, Spuren zu hinterlassen, die von nachfolgenden Projekten übernommen werden können. Ein nachhaltiges Projekt wäre demnach eines, das die Erfahrung und die Fähigkeit seiner lokalen Verwalter und seiner Begünstigten, andere Projekte besser einzuführen oder zu verwalten, erhöht hat, auch wenn diese vom ersten Projekt völlig verschieden sind.

$\checkmark$ Es gibt wenig westliche NRO, die sich selbst finanzieren; die meisten, wenn nicht alle, erhalten zum Überleben Mittel aus staatlichen Subventionen oder aus privaten Spenden. Dies ist besonders der Fall bei den öffentlichen digitalen Zugangsstellen, die von Verbänden oder kommunalen Körperschaften abhängen. Daher kann man in Afrika nicht Ergebnisse erwarten, die von westlichen Institutionen nicht erzielt werden. Die niedrigen Budgets der - übrigens kurzfristigen - Mittel für die Entwicklungszusammenarbeit sind im Allgemeinen unzureichend für die erwartete gesellschaftliche Aneignung, da diese eine genügend lange Zeit und somit bedeutendere Mittel erfordert. 


\section{$\square$ Der Fernunterricht durch die IKT}

Die Entwicklungszusammenarbeit steht, indem sie den Fernunterricht durch die IKT zu einem weiteren ihrer Anliegen macht, vor lokalen Zwängen, die mit der Infrastruktur, der Ausrüstung und vor allem mit dem Bildungskonzept, das heisst mit Inhalt, Methodik und Pädagogik grosser Gruppen von Lernenden zusammenhängen. Das Projekt der afrikanischen virtuellen Universität und jenes der französischsprachigen virtuellen Universität, die in den Räumlichkeiten der Weltbank bzw. der Frankophonie konzipiert wurden, sind zwar attraktiv, bleiben aber bislang elitistisch, klientelistisch und ohne Beziehung zum lokalen Bildungssystem. Nur Länder, lokale Organe, Sektoren und Tochtergesellschaften, die in der Gunst dieser Institutionen stehen, kommen zur Auswahl, ohne dass sie zwangsläufig am geeignetsten dafür sind, und noch bevor sie eine Politik festgelegt haben, die fähig ist, den neuen Unterricht aufzuwerten, die Nachhaltigkeit des Projekts zu gewährleisten und dieses mit dem bestehenden Bildungssektor $\mathrm{zu}$ verbinden. Das auf die afrikanischen Entscheidungsträger ausgerichtete GDLN-Konzept der Weltbank ist ein Beispiel dafür und dient vielmehr der örtlichen Neugier, als der Hebung des Unterrichtsniveaus. Afrika wird somit zum Theater von „Schaukastenprojekten“, deren lokale ,Verantwortliche“, die während der „Pilotzeit" Tagegeld erhalten, sich kaum in den lokalen Bildungsstrukturen wiedererkennen und in der Angst des zeitlich befristeten Vertrags leben.

\section{Schlussfolgerung}

Die kritischen Gesichtspunkte in der Praxis der internationalen Zusammenarbeit im IKT-Bereich, die wir in dieser Studie dargelegt haben, bringen uns zur Schlussfolgerung, dass man sich eine andere Art der Zusammenarbeit vorstellen muss, ausgehend von einer anderen Art, den lokalen IKT-Bedarf zu erfassen.

Ein solcher Ansatz sieht den afrikanischen IKT-Bedarf und die Notwendigkeit der Zusammenarbeit in diesem Bereich gemäss der erlebten Erfahrung der Unterentwicklung vor. Er versucht, diesen Bedarf im lokalen Umfeld zu definieren, das heisst nach dem, was - diesmal - die lokalen Partner gegenüber den Informations- und Kommunikationstechnologien empfinden; was den Elan für diese neuen Technologien fördert und/oder hemmt; was erforderlich ist oder was für die Afrikaner, die mit den IKT in Kontakt sind, einen Sinn hat; nach der Hilfe, welche Afrika zur Umorientierung der Aktionen des Nordens für eine neu überdachte Partnerschaft und eine verantwortungsvolle Übernahme der gegenseitigen Herausforderungen erbringen kann. Diese Konzeption kann zwar die Gewohnheiten von weniger aufgeschlossenen Geldgebern ändern, jedoch wird sie das Verschwinden der Missionarsideologie und der hegemonischen Haltungen einleiten, die für die von der Entwicklungszusammenarbeit gewünschten „Mitentwicklung“ wenig hilfreich sind.

Desgleichen nimmt dieser Vorschlag den Vorgang der Integration und der gesellschaftlichen Aneignung der IKT als eine ausgehandelte Hypothese, die jeder Planung vorausgehen würde - kurz gesagt, als eine durch die Situation bestimmte Dynamik - wahr. So soll das, was als Problem oder besser gesagt als Hindernis 
wahrgenommen wird, zu einer Gelegenheit werden, die es auszuwerten gilt, da dies dazu anregt, die Quelle des lokalen Widerstands zu identifizieren und die eingeleitete Dynamik zu überprüfen. Das Budget des Projekts wird, obwohl es festgesetzt ist, zum Gegenstand ständiger Verhandlung. Fortan wird der Schwerpunkt auf die Initiativen der lokalen Akteure gelegt, die man - diesmal - als Partner betrachtet, da man, indem man sie schult, sich selbst anhand der Situationen vor Ort schult. Die Evaluation des Projekts zur Einführung der IKT berücksichtigt somit als Kriterien den Beteiligungsgrad, die lokale Fähigkeit zur gesellschaftlichen Aneignung des Projekts und die wieder zu verwendende Wissensdividende. Sie erfolgt im Rahmen einer langfristigen Sicht.

Wenn es eine der derzeitigen Herausforderungen der Informationsgesellschaft im Allgemeinen und der IKT im Besonderen ist, Schnittstellen und Dienstleistungen dem afrikanischen Umfeld anzupassen, die Gemeinschaften in angemessener Weise untereinander und an die ganze Welt anzuschliessen, die übermässigen Kosten der Struktureinrichtungen und ihrer Nutzung zu senken, den Grad der endogenen Kontrolle der Bevölkerung über die IKT zu erhöhen, den Kommunikationsrhythmus zu beschleunigen, um Wirtschaft und Kultur zu dynamisieren, dann ist man gezwungen, die Frage der Zusammenarbeit im IKT-Bereich von einem Standpunkt des ständigen Dialogs zwischen dem Norden und dem Süden aus zu überdenken, bei dem die Erfahrungen der Beteiligten im Verlauf ihrer Gegenüberstellung kontinuierlich neu bewertet werden. Dies ist eine der Voraussetzungen dafür, dass sich die von der Entwicklungszusammenarbeit gewünschten Netzwerke im IKT-Bereich effizient in die Bemühung Afrikas für eine nachhaltige Entwicklung integrieren, und dass die Zusammenarbeit zu ihrem richtigen Wert evaluiert wird.

Bei dieser Wahrnehmung ist die Förderung der Forschung wesentlich. Wenn nämlich mehrere Projekte der Entwicklungszusammenarbeit den Schwerpunkt auf die Aneignung der IKT und die ,gesellschaftliche Beherrschung“ des Internets legen, so geht es im einen oder anderen Fall vor allem um die verschiedenen Nutzungen der IKT für den Entwicklungsbedarf; es geht um den Lernprozess für die Handhabung der Internet-Werkzeuge, die Fähigkeit zum Heraufladen des afrikanischen Inhalts auf das Web, die Kompetenz für die Anwendungen der Technologien in Afrika. Diese Bedeutung der Aneignung lag der Mehrheit der Projekte zur Ausdehnung der IKT und zur Schulung im IKTBereich zugrunde und trägt sie auch weiterhin. Nun sollte aber die Aneignung des Internets in Afrika so verstanden werden, dass man sich zwar eine gründliche Kenntnis der Nutzung, aber auch die Fähigkeit zur Konzeption und Gestaltung der Ideen und der Internet-Werkzeuge verschafft. Dann würde es darum gehen, in Afrika ein Klima ständigen Forschens und Ausprobierens zu schaffen, das für ein Streben nach Aneignung in Form von Beteiligung unerlässlich ist.

Indessen hat der drastische Rückgang der öffentlichen Entwicklungshilfe seit Mitte der 90er Jahre vor allem den Forschungsbereich in Afrika getroffen. Es obliegt fortan einer nachhaltigen Entwicklungszusammenarbeit und einer verantwortlichen Partnerschaft, das Konzept „lokale Entwicklung ohne lokale Forscher" zu vergessen, denn es besteht effektiv ein Bedarf an lokaler Forschung im IKT-Bereich. Es könnte sich unter anderem um die Datenverarbeitung der 
afrikanischen Sprachen ${ }^{44}$, um die auditive Darstellung der gesprochenen Sprachen in den Wissensdatenbanken, um die Funktionsweise der zu ihrer Integration geeigneten Software, um die Formalisierung gewisser Logiken der afrikanischen Rede zum Zweck ihrer Software-Umsetzung ${ }^{45}$, um die Denkweisen einer Gruppe von Menschen, die von Sprachtypen bestimmt sind, welche anderen Logiken als denen der Digitalisierung gehorchen, um die Artikulation der Einstufungen bei der lokalen Beherrschung der $\mathrm{IKT}^{46}$, um die Infragestellung der „Entwicklung“ durch Projekte an sich, usw. handeln. Die Forschung ist der Bereich, in dem die Entwicklungszusammenarbeit eine wesentliche Rolle spielen sollte. Die Aufbringung der Ressourcen und der Austausch von Wissen, Kompetenzen und auch Finanzmitteln sind für die digitale Innovation, die gesellschaftliche Beherrschung der neuen Technologien sowie für die Optimierung der menschlichen und institutionellen Kapazitäten unerlässlich. Jedoch ist dieser Aufruf an die internationale Hilfe nicht im leidenschaftlichen Ausdruck unilateraler Unterstützung, blosser Nutzung der Bemühungen der anderen, bedingungsloser Einbringung von frischem Kapital, usw. zu sehen. Es geht vor allem um die Heranziehung der Erfahrung der anderen mit dem Ziel, Widerstände gegen die Neuerung zu brechen; um die Infragestellung dieser Erfahrung zum Zweck, eine kontextbezogene Forschung einzuleiten; um die gemeinsame Bewältigung der Probleme für eine gerechte Bewirtschaftung der geistigen und gewerblichen Eigentumsrechte; um gemeinsame Anstrengungen zur Senkung der Kosten bei der Herstellung und dem Konsum der neuen Technologien, usw. Die Wiedereröffnung und/oder Errichtung von Forschungszentren im IKT-Bereich wird unter diesem Gesichtspunkt zu einem Grundbedarf, der sowohl auf der Ebene der Staaten als auch der Unternehmen prioritär ist und aufhört, gemäss einer gewissen Entwicklungszusammenarbeit, für Afrika ein Luxus zu sein.

44 Siehe das Forschungsprogramm von UMR Nr. 8135, „Langage, langues et cultures d'Afrique noire“ (LLACAN), unter der Zuständigkeit des CNRS, von INALCO und der Universität Paris VII, das die Datenverarbeitung der afrikanischen Sprachen zum Thema hat: „Le traitement informatique des langues africaines" <http://llacan.cnrs-bellevue.fr $>$.

45 Wir denken hier an bestimmte Argumentationsgewohnheiten in Afrika, die durch die argumentative Inversion, die anaphorische Rückweisung auf den positiven Satz des Weltallbildes, usw. geprägt sind. Siehe R. Ntambue-Tshimbulu, La logique formelle en Afrique noire. Problématique, enseignement et essais, Louvain-la-Neuve, Academia-Bruylant, 1997.

46 Vgl. A. Chéneau-Loquay, „Les relations entre l'Etat, le droit et les réseaux techniques sont-elles obligatoires dans le processus de modernisation en Afrique? Réflexions à partir du cas africain“, Terminal, Nr. 84, Frühjahr 2001, <www.africanti.org/resultats/documents/ACLTerminal1.PDF>. 


\section{Bibliographie}

\section{Bücher und Unterlagen}

Analysis, The Network Revolution and the Developing World, Final Report for World Bank and InfoDev, Analysis Report Nr. 00-216, 17. August 2000, <www.infodev.org/ projects/internet/400networkingrevolution/ 400.pdf>.

Assemblée nationale, Rapport d'information déposé par la Commission des finances, de l'économie générale et du plan sur les activités et le contrôle du Fonds monétaire international et de la Banque mondiale présenté par M. Yves Tavernier, député, 12. Dezember 2000.

Brunet, P.J., Tiemtoré, O., Vettraino-soulard, M.-C., Les enjeux éthiques d'Internet en Afrique de l'Ouest. Vers un modèle éthique d'intégration, Paris, L'Harmattan, 2002.

Buyck, B., Emploi par la Banque de l'assistance technique pour le développement institutionnel, document de travail des services de la Banque mondiale Nr. 578, Washington, D.C., BIRD, 1991.

Chéneau-Loquay, A., Enjeux des technologies de la communication en Afrique. Du téléphone à Internet, Paris, Regards; Karthala, 2000.

Cogburn, D.L., Nyaki Adeya, C., Exploring the Challenges and Opportunities for Africa in the Information Economy, Discussion Paper Series, Nr. 2000-3, Maastricht, UNU/INTECH, 2000, $<w w w . i n t e c h . u n u . e d u / p u b l i c a t i o n s / d i s c u s s i o n-p a p e r s / 2000-3 . p d f>$.

Conquy Beer-Gabel, J., Informatisation du tiers-monde et coopération internationale, Paris, La Documentation Française, 1984.

Dutch Development Cooperation, The Development Divide in a Digital Age: A Strategy for Dutch Development Cooperation, 2000.

Gaillard, J., La coopération scientifique et technique avec les pays du Sud, coll. Homme et Société, Paris, Karthala, 1999.

G-8 (2001-2002), A New African Initiative: Merger of the Millennium Partnership for the African Recovery Programme (MAP) and Omega Plan, einsehbar unter <www.g8.gc.ca>.

—, Plan d'action pour l'Afrique, <www.g8.gc.ca/2002Kananaskis/kananaskis/afraction-fr.pdf>.

HCCI, Biens publics mondiaux et coopération internationale. Nouvelle stratégie pour de nouveaux enjeux, Paris, Karthala, 2002.

Kaul, I., Grunberg, I., Stern, M.A. (dir.), Les biens publics à l'échelle mondiale. La coopération internationale au XXI siècle, Oxford, Oxford University Press, 1999.

Michel, J.A., Coopération pour le développement. Efforts et politiques des membres du Comité d'aide au développement. Rapport du président du Comité d'aide au développement. Edition 1999, Paris, OCDE, 1999.

Ntambue-Tshimbulu, R., La logique formelle en Afrique noire. Problématique, enseignement et essais, Louvain-la-Neuve, Bruylant Academia, 1997.

-, L'Internet, son web et son e-mail en Afrique. Approche critique, coll. Etudes africaines, Paris, L'Harmattan, 2001.

Nulens, G., Hafkin, N., Van Audenhove, I., Cammaerts (eds.), The Digital Divide in Developing Countries: Towards an Information Society in Africa, Brussels, VUB University Press, 2001.

Ossama, Fr., Les nouvelles technologies de l'information. Enjeux pour l'Afrique subsaharienne, Paris, L'Harmattan, 2001.

Rathgeber, M., Ofwona Adera, E. (dir.), L'inégalité des sexes et la révolution de l'information en Afrique, CRDI, 2000, $280 \mathrm{~S}$. , <www.idrc.ca/books/focus/941/01-front.html>.

Rist, G. (dir), Les mots du pouvoir, sens et non-sens de la rhétorique internationale, Nouveaux Cahiers de l'iuéd, Nr. 13, Genf, iuéd; Paris, PUF, 2003.

Soupizet, J.-F., Gille, L. (dir.), Nord et Sud numériques, Les Cahiers du numérique, Band 2, Nr. 3-4, 2001.

UNDP, Technical Cooperation in African Development: An Assessment of Its Effectiveness on Support of the UN Program of Action for African Economic Recovery and Development, 1986-1990, PANUREDA, Washington D.C., 1990.

World Bank, Information and Communication Technologies (ICT), Sector Strategy Paper, 2002.

\section{Artikel}

Bernard, E., „Académies Cisco en Afrique de l'Ouest: quel est le jeu des institutions internationales ?“, Januar 2001, <www.accessit.org/bibliotheque/dossiers/pnud_cisco.htm>.

Blanchard, J.-M., „De la pertinence de l'usage de l'Internet dans les pays en voie de développement“, in Soupizet, J.-F., Gille, L. (dir.), Nord et Sud numériques, Les Cahiers du numérique, Band. 2, Nr. 34, 2001. 
Chéneau-Loquay, A., „Les relations entre l'Etat, le droit et les réseaux techniques sont-elles obligatoires dans le processus de modernisation en Afrique? Réflexions à partir du cas africain“, Terminal, Nr. 84, Frühjahr 2001, <www.africanti.org/resultats/documents/ACLTerminal1.PDF>.

Crozet, M.-P., Schmid, D., „L'approche sectorielle: une nouvelle modalité de l'aide européenne au développement“, Afrique contemporaine, Nr. 193, 1. Quartal 2000, S. 76-89.

Evans, P., ,The Eclipse of the State?“, World Politics, Baltimore, Johns Hopkins University Press, Oktober 1997, S. 63.

Gaillard, J., Waast, R., „Quelles politiques de coopération scientifique et technique avec l'Afrique?“, Afrique contemporaine, Sondernummer, 4. Quartal 1998, S. 85-97.

Gaulme, Fr., „Les nouvelles orientations de l'aide publique au développement“, Afrique contemporaine, Sondernummer, 4. Quartal 1998, S. 101-118.

Heller, P.S., Gupta, S., „Les enjeux de l'expansion des flux d'aide“, Finances et développement (Vierteljahreszeitschrift des IWF), Juni 2002, <www.imf.org/pubs/ft/fandd/fre/2002/06>.

Humbaire, B. „L'Internet et l'Afrique. Ressources documentaires“, Afrique contemporaine, Nr. 182, 2. Quartal 1997.

Levy, M., „Gouttes d'huile ou grains de sable“, in Voyage dans la galaxie des ONG. Actes du colloque sur les ONG et leur rôle dans la coopération au développement, Brüssel, 2. Oktober 1999, Demain le monde, Januar 2000, S. 4.

Renaud, P., „Un partenariat qui suscite des interrogations. Point de vue sur la coopération CISCOPNUD pour la formation d'Africains aux NTIC“, Auszug aus der Liste Africa'nti, 5. Juni 2001, unter <www.africanti.org $>$. Ebenfalls veröffentlicht in Elie M. (dir.), Le fossé numérique. L'Internet, facteur de nouvelles inégalités?, Problèmes politiques et sociaux, Nr. 861, 10. August 2001, S. 5859.

Severino, J.-M., „Les fondements de l'aide au développement au XXI ${ }^{\mathrm{e}}$ siècle“, Critique internationale, Januar 2001.

Fukuda-Parr, S., „Mettre les nouvelles technologies au service du développement humain“, Präsentation des UNDP-Berichts 2001, in HCCI, Biens publics mondiaux et coopération internationale. Nouvelle stratégie pour de nouveaux enjeux, Paris, Karthala, 2002, S. 96-107.

\section{Internet-Adressen}

Africa'nti, observatoire des modes d'insertion, des usages et des impacts des TIC en Afrique et dans les Suds : <www.africanti.org >.

AISI (African Information Society Initiative) : <www.uneca.org/aisi $>$.

Bellanet, collaboration pour le développement par l'usage des TIC : <www.bellanet.org $>$.

CEA (UN-Wirtschaftskommission für Afrika) : <www.uneca.org $>$.

CTO (Commonwealth Telecommunications Organisation) : <www.cto.com $>$.

DFID, Regierungsstelle für Entwicklungszusammenarbeit, Grossbritannien : <www.dfid.gov.uk>.

Europäische Kommission, institutionelle Aspekte der europäischen Politiken betreffend die Informationsgesellschaft : <http://europa.eu.int/information_society/index_en.htm>.

Famafrique, Website über Initiativen betreffend Frauen : <www.famafrique.org/nouv/nouv.html $>$.

Fondation du devenir, réseau Anaïs : <www.anais.org $>$.

IDRC, Forschungszentrum für internationale Entwicklung, Kanada, Kooperationsprogramm im IKTBereich mit Afrika: <www.idrc.ca>.

InfoDev (The Information for Development Program) der Weltbank, zahlreiche Publikationen zur Zusammenarbeit im IKT-Bereich: <www.infodev.org >.

Initiative Acacia für Afrika des Forschungszentrums für internationale Entwicklung (CRDI) Kanada: $<$ www.idrc.ca/acacia/acacia_f.htm >.

Initiative GDLN (Global Development Learning Network) der Weltbank, IKT für die Entwicklungsausbildung: <www.gdln.org/french/Index.htm>.

Initiative Leland für Afrika: <www.usaid.gov/regions/afr/leland>.

INTIF (Institut francophone des nouvelles technologies de l'information et de la formation) : $<$ http://intif.francophone.org $>$.

NEPAD (Neue Partnerschaft für die Entwicklung Afrikas), IKT-Bereich : <www.nepad.org>.

ONU, Observatoire des Nations unies des technologies de l'information:

$<$ www.sdnp.undp.org/observatory $>$.

PICTA (Partenariat pour les technologies de l'information et de la communication en Afrique) : <www. uneca.org/aisi>.

RASCOM (Regional African Satellite Communications Organisation) : <www.dfa.gov.za/for-relations/ multilateral/rascom.htm>. 
Regionale afrikanische Vorbereitungskonferenz auf den Weltgipfel zur Informationsgesellschaft: $<$ http://www.geneva2003.org/bamako2002>.

Synergie Gender und Entwicklung, IKT- und Gender-Programme : <www.enda.sn/synfev/synfev.htm>.

TICAD, Internationale Konferenz in Tokio über die Entwicklung Afrikas, Zusammenarbeit zwischen Afrika und Asien im IKT-Bereich: <www.ticad.gouv.bj>.

UNDP und ORBICOM (2002), Observatoire de la fracture numérique: <www.orbicom.uqam.ca/projects/ ddi2002>.

UNITAR, (Ausbildungs- und Forschungsinstitut der Vereinten Nationen) : <www.unitar.org $>$.

UNO, UN ICT Task Force : <www.un.org >.

World Computer Exchange, NRO zum Sammeln von EDV-Gebrauchtmaterial für den Süden: $<$ www.worldcomputerexchange.org $>$. 